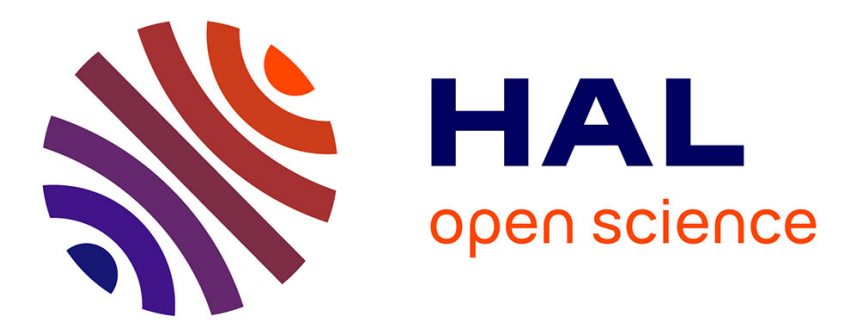

\title{
L'industrie lithique épigravettienne de Saint-Antoine - Locus 2 (Vitrolles, Hautes-Alpes) : première analyse
}

Jean-Pierre Bracco, Jean Gagnepain, Christian Stouvenot, Patrick Bidart, Serge Vigier

\section{- To cite this version:}

Jean-Pierre Bracco, Jean Gagnepain, Christian Stouvenot, Patrick Bidart, Serge Vigier. L'industrie lithique épigravettienne de Saint-Antoine - Locus 2 (Vitrolles, Hautes-Alpes): première analyse. PALEO : Revue d'Archéologie Préhistorique, 1997, 9, pp.221 - 243. 10.3406/pal.1997.1234 . hal03095768

\section{HAL Id: hal-03095768 \\ https://hal.science/hal-03095768}

Submitted on 4 Jan 2021

HAL is a multi-disciplinary open access archive for the deposit and dissemination of scientific research documents, whether they are published or not. The documents may come from teaching and research institutions in France or abroad, or from public or private research centers.
L'archive ouverte pluridisciplinaire HAL, est destinée au dépôt et à la diffusion de documents scientifiques de niveau recherche, publiés ou non, émanant des établissements d'enseignement et de recherche français ou étrangers, des laboratoires publics ou privés. 


\section{L'industrie lithique épigravettienne de Saint-Antoine - Locus 2} (Vitrolles, Hautes-Alpes) : première analyse Jean-Pierre Bracco, Jean Gagnepain, Christian Stouvenot, Patrick Bidart, Serge Vigier

\section{Citer ce document / Cite this document :}

Bracco Jean-Pierre, Gagnepain Jean, Stouvenot Christian, Bidart Patrick, Vigier Serge. L'industrie lithique épigravettienne de Saint-Antoine - Locus 2 (Vitrolles, Hautes-Alpes) : première analyse. In: Paléo. N. 9,1997. pp. 221-243;

doi : https://doi.org/10.3406/pal.1997.1234

https://www.persee.fr/doc/pal_1145-3370_1997_num_9_1_1234

Fichier pdf généré le 24/05/2018 


\title{
Résumé
}

La fouille préventive du locus 2 du gisement de Saint-Antoine (Vitrolles, Hautes-Alpes), dans les Alpes du sud, a livré une importante série lithique épigravettienne. La zone principale d'approvisionnement en silex est distante de plus de $30 \mathrm{~km}$ du site (moyenne vallée de la Durance). Le schéma opératoire est orienté vers la production de lamelles rectihgnes et leur transformation en armatures à dos. L'ensemble du débitage s'effectue au percuteur de pierre tendre. Typologiquement, la présence de nombreuses pointes à dos rectihgne, surtout de type microgravettes et de lamelles à bord abattu, l'absence de géométriques et de la technique du microburin, autorisent un rapprochement avec l'Epigravettien évolué ou final des séries italiennes.

\begin{abstract}
The salvage excavation of locus 2 of the site of Saint-Antoine (Vitrolles, Hautes-Alpes), located in the southern part of the French Alps, has yieldied an abundant Epigravettian hthic assemblage. The principal flint procurement zone is situated more than $30 \mathrm{~km}$ south of the site. Reduction sequence is oriented toward the production of rectilinear bladelets and their eventual transformation into backed projectile points. The debitage is effectuated by the use of soft stone hammers. Typologically, the presence of numerous rectilinear backed points, espacially microgravettes and backed bladelets, along with the absence of geometric tools and the microburin technique, permit a correlation to be made with the evolved or final Epigravettian of some Italian assemblages.
\end{abstract}




\title{
L'INDUSTRIE LITHIQUE ÉPIGRAVETTIENNE DE SAINT-ANTOINE - LOCUS 2 (VITROLLES, Hautes-Alpes) : PREMIÈRE ANALYSE
}

\author{
Jean-Pierre BRACCO(1), Jean GAGNEPAIN(2), Christian STOUVENOT(3), \\ Patrick BIDART ${ }^{(4)}$ et Serge VIGIER ${ }^{(5)}$
}

Résumé : La fouille préventive du locus 2 du gisement de Saint-Antoine (Vitrolles, Hautes-Alpes), dans les Alpes du sud, a livré une importante série lithique épigravettienne. La zone principale d'approvisionnement en silex est distante de plus de $30 \mathrm{~km}$ du site (moyenne vallée de la Durance). Le schéma opératoire est orienté vers la production de lamelles rectilignes et leur transformation en armatures à dos. L'ensemble du débitage s'effectue au percuteur de pierre tendre. Typologiquement, la présence de nombreuses pointes à dos rectiligne, surtout de type microgravettes et de lamelles à bord abattu, l'absence de géométriques et de la technique du microburin, autorisent un rapprochement avec l'Epigravettien évolué ou final des séries italiennes.

Mots-clés : Epigravettien, Alpes du sud, matières premières minérales, technologie lithique, typologie lithique, armatures à dos.

Abstract : The salvage excavation of locus 2 of the site of Saint-Antoine (Vitrolles, Hautes-Alpes), located in the southern part of the French Alps, has yieldied an abundant Epigravettian lithic assemblage. The principal flint procurement zone is situated more than $30 \mathrm{~km}$ south of the site. Reduction sequence is oriented toward the production of rectilinear bladelets and their eventual transformation into backed projectile points. The debitage is effectuated by the use of soft stone hammers. Typologically, the presence of numerous rectilinear backed points, espacially microgravettes and backed bladelets, along with the absence of geometric tools and the microburin technique, permit a correlation to be made with the evolved or final Epigravettian of some Italian assemblages.

Key-words : Epigravettian, lithic raw material, lithic technology, lithic typology, backed points.

La butte marneuse de Saint-Antoine (Vitrolles, HautesAlpes) se trouve en rive droite de la Durance, entre Sisteron et Gap, à 575 mètres d'altitude (fig. 1). Dominée par le Pic de Crigne (1262 m), cette région s'apparente à un milieu accidenté de moyenne montagne, marqué par une forte empreinte glaciaire.

Une première occupation épigravettienne de plein air, d'environ $50 \mathrm{~m}^{2}$, avait été reconnue et partiellement fouillée de 1988 à 1990. Sur la base de l'industrie lithique et en l'absence de résultats radiométriques cohérents, le gisement fut rapporté à un épigravettien final typologiquement proche des séries tardiglaciaires transalpines (Muret et al., 1991).

Dans le cadre des travaux de construction de l'Autoroute A51, secteur Sisteron-La Saulce, une opération préventive pilotée par l'AFAN fut organisée. Les objectifs étaient d'une part de fouiller les zones périphériques du gisement et d'autre part de réaliser une série de tranchées

(1) Jean-Pierre Bracco, Maison Méditerranéenne des Sciences de l'Homme, LAPMO-URA 164, 5, rue du Château de I'Horloge, BP 647, 13094 Aix-en-Provence Cedex 2 et AFAN

(2) Jean Gagnepain, UMR 9948, Place de la Mairie, 04500 Quinson et AFAN

(3) Christian Stouvenot, AFAN, 17, rue Hanappier, 33200 Bordeaux

(4) Patrick Bidart, AFAN, 29, rue Poissonnerie, 64100 Bayonne

(5) Serge Vigier, AFAN, 22, rue Chai des Farines, 33000 Bordeaux 


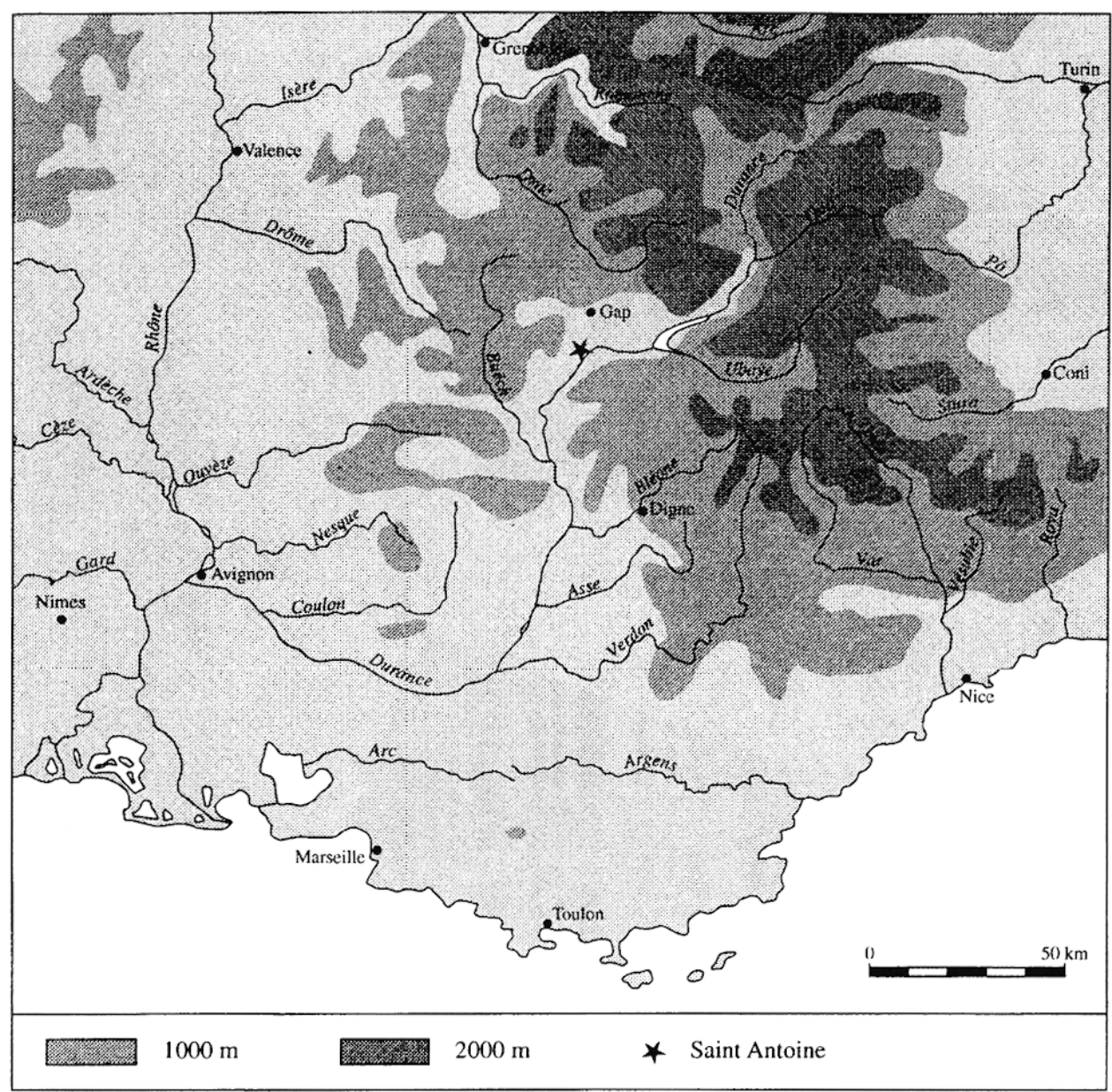

Figure 1 : Saint-Antoine à

Vitrolle.

Localisation du site

Site location

de reconnaissance afin de reconnaître d'éventuelles extensions de l'occupation. Ces tranchées ont mis en évidence un second site épigravettien, à une dizaine de mètres au sud-est du premier (et nommé locus 2, le premier site devenant alors le locus 1). Simultanément, des travaux de décapages exécutés sur la même butte par une autre équipe $A F A N^{(6)}$ pour le repérage de structures néolithiques, mirent également en évidence une troisième occupation du Paléolithique supérieur final, d'une surface estimée de $2500 \mathrm{~m}^{2}$ (locus 3).

Les fouilles n'ont concerné que les locus 1 et 2 . Le locus 3 , non directement menacé par les travaux autoroutiers, a été soumis à prescription archéologique. Une premiè- re intervention de terrain s'est déroulée de Septembre à Décembre 1995 et a concerné la fin de la fouille du locus 1 et la réalisation de sondages complémentaires (Gagnepain et Bracco, 1996). Une deuxième opération, consacrée à la fouille du locus 2, s'est déroulée de Février à Juin $1996^{(7)}$ (Gagnepain et al., 1997). C'est l'industrie de ce dernier site, fouillé extensivement en une seule campagne, qui sera ici présentée.

\section{Le contexte archéologique}

La fouille du locus 2 à porté sur environ $100 \mathrm{~m}^{2}$ pour une surface totale estimée à $120 \mathrm{~m}^{2}$. Deux niveaux archéologiques principaux ${ }^{(8)}$, inclus dans un limon argileux mis en

(6) L'équipe «néolithique» était dirigée par Noël Coye. Il nous est agréable d'en remercier ici tous ses membres pour leur collaboration dans la découverte et le diagnostic du locus 3.

(7) La fouille du locus 2 a été organisée sous la coordination de J. Gagnepain, responsable d'opération, J.P. Bracco, traitement de l'industrie lithique, $P$. Bidart et $S$. Vigier, responsables de secteur.

(8) Un troisième niveau inférieur, non identifié à la fouille mais clairement mis en évidence par les projections, est présent dans certaines zones du site (analyse de A. Canals i Salomo, UMR 9948, Laboratoire de Préhistoire du Lazaret). Son interprétation reste encore indécise : réel niveau anthropique ou «décrochage» de pièces provenant du niveau $B$. 
place par colluvionnement, ont pu être individualisés ${ }^{(9)}$. Le niveau supérieur est pauvre en industrie lithique. Les restes osseux ne sont présents qu'à l'état de fragments très érodés et aucune structuration claire de l'espace n'a pu être mise en évidence excepté la présence d'un foyer construit (Gagnepain et al., 1997). Le niveau inférieur est épais de $50 \mathrm{~cm}$ en moyenne. Très riche en industrie lithique, il a livré plusieurs indices d'une organisation de l'espace sous la forme d'associations préférentielles de matières premières minérales ou de catégories d'artefacts (Canals i Salomo, 1997). Mais la structure la plus évidente est constituée par la présence d'un grand amas d'ossements formé de plusieurs centaines d'éléments osseux (Cervus elaphus : 94,5\% NRD, Bos sp. : $5,5 \%$ NRD). Les premiers résultats de l'analyse archéozoologique suggèrent, pour les cervidés, une représentation partielle des carcasses (absence du squelette axial), l'absence d'individus très jeunes ou très âgés et une période d'abattage probable entre Septembre et Novembre ${ }^{(10)}$.

Cette importance des restes osseux peut être mise en parallèle avec les premiers résultats de l'analyse fonctionnelle de l'industrie lithique qui indiquent d'importantes activités de boucherie et de traitement des peaux (Philibert, 1997).

En l'état actuel, l'interprétation proposée pour le gisement de Saint-Antoine est celle d'une succession d'occupations très spécialisées liées à la chasse des cervidés et au traitement des carcasses.

\section{L'Industrie lithique : une première approche typo- technologique}

\section{II.1 Conditions de l'étude}

Compte tenu des délais disponibles et du volume à traiter, la fouille a été effectuée par quart de mètre carré et par passées de décapage de 3 à $5 \mathrm{~cm}$. L'ensemble des sédiments à été tamisé. II n'a pas été possible dans un premier temps de séparer les pièces lithiques selon les 2 niveaux et l'analyse présentée ici sera donc globale.

La série est constituée de plusieurs dizaines de milliers d'artefacts. Dès le stade de la fouille, le matériel lithique a été séparé en deux lots :

- le premier est constitué de toutes les pièces inférieures à $1 \mathrm{~cm}$, pour la plupart retrouvées au tamisage. Pour ce lot, formé de 57780 pièces, seul un décompte du nombre de pièces par unité de volume fouillée (quart de $\mathrm{m}^{2} /$ décapage) a été réalisé. Ces données ont été utilisées pour l'analyse de la répartition spatiale du matériel (Gagnepain et al., 1997) ;

- le deuxième lot comprend la totalité des pièces d'une longueur maximale supérieure à $1 \mathrm{~cm}$, augmenté de toutes les pièces retouchées quelle que soit leur taille. II comprend 13596 pièces et c'est à partir de cette série qu'ont été effectués les décomptes et analyses.

L'étude s'appuie essentiellement sur la lecture technologique des pièces. Faute de temps, les remontages n'ont pu faire l'objet que de quelques tests préliminaires.

\section{II.2 Présentation générale}

\section{État général de la série}

L'industrie est en général bien préservée. Les pièces patinées sont peu abondantes $(7,2 \%)$, les tranchants sont en bon état, sans traces importantes d'esquillement ou de concassage. Toutefois, le taux de fracturation est très élevé $(74,3 \%)$. II semble principalement lié en général à des mouvements de compression-décompression à l'intérieur du sédiment. Des fractures d'utilisation sont aussi déterminables sur les armatures à dos. Enfin, de très nombreuses pièces sont brûlées (39\%), entraînant pour de nombreux objets uné difficulté de reconnaissance de la matière première et des stigmates.

\section{Nature de l'assemblage}

Un premier décompte général de l'ensemble du matériel lithique permet de souligner plusieurs points (tableau 1):

\begin{tabular}{lrr}
\hline & fréquence & $\%$ \\
\hline éclats indéterminés & 984 & 7,2 \\
lames et lamelles & 6733 & 49,5 \\
dont entières & $(1027)$ & $(15,3)$ \\
dont cassées & $(5706)$ & $(84,7)$ \\
sous-produits (sur lame ou éclats) & 1220 & 9 \\
produits à crête & 28 & 0,2 \\
nucléus & 303 & 2,2 \\
dont laminaires & $(301)$ & \\
dont à éclats & $(2)$ & \\
pièces indéterminées & 4328 & 31,8 \\
Total & 13596 & \\
\hline
\end{tabular}

Tableau 1 : Saint-Antoine - locus 2

Décompte de l'industrie lithique

Lithic industry counting

- le nombre de pièces indéterminées est élevé $(\mathrm{N}=4328$ soit $31,8 \%$ ), en raison de la fracturation du matériel et de sa rubéfaction ;

- l'objectif premier du débitage apparaît clairement : les supports laminaires ou lamellaires constituent la moitié des artefacts retrouvés et presque $90 \%$ des supports de plein débitage $(87,2 \%$ du total lames + éclats). Même en tenant compte de leur fort taux de fracturation $(84,7 \%)$ et donc d'une sur-représentation probable au niveau des décomptes, ils restent en tout état de cause très dominants ;

(9) Les analyses sédimentologiques et géomorphologiques ont été réalisées par P. Bertran (UMR 9933, Bordeaux et AFAN), C. Jorda (AFAN) et M. Jorda (URA 903, Université de Provence).

(10) Etude effectuée par J.F. Bez, URA 164 - LAPMO. 
- Les sous-produits de débitage sont faiblement représentés, surtout les produits à crête. Cette sous-représentation est surprenante au regard du nombre de nucléus, qui sont abondants $(N=303)$. Elle ne semble donc pas a priori être liée à l'absence d'activités de taille sur le site.

\section{Principaux caractères de l'outillage retouché}

L'outillage retouché est dominé par le groupe des armatures à dos qui représente plus de $90 \%$ de l'ensemble des outils (tableau 2). Les autres outils sont très peu nombreux à l'exception des grattoirs.

\begin{tabular}{lrr}
\hline & fréquence & $\%$ \\
\hline burins & 5 & 0,2 \\
grattoirs & 199 & 7,5 \\
pièces esquillées & 12 & 0,4 \\
pièces à encoches & 9 & 0,3 \\
pièces tronquées & 7 & 0,3 \\
pièces à retouches abruptes & 17 & 0,6 \\
pièces à retouches discontinues & 9 & 0,3 \\
armatures à dos & 2413 & 90,3 \\
Total & $\mathbf{2 6 7 1}$ & \\
\hline
\end{tabular}

Tableau 2 : Saint-Antoine - locus 2

Décompte de l'outillage retouché

Retouched tools counting

\section{II.3 Les matières premières minérales}

Le silex est la seule matière première utilisée pour le débitage, si l'on excepte quelques rares éclats en quartz $(\mathrm{N}=7)$.

\section{L'approvisionnement en silex}

Plusieurs variétés de silex sont représentées dans la série du locus 2 (fig. 2). Cinq groupes principaux ont été distingués (pour une description plus précise des matières premières siliceuses et des sources d'approvisionnement cf. Stouvenot, 1997) :

- silex bédoulien : c'est la variété la plus abondante $(N=$ 8858 : 65,2\%). II s'agit d'un silex gris clair à noir, parfois zoné ou tacheté. Le gîte le plus proche est situé à vol d'oiseau à environ $30 \mathrm{~km}$ en aval du site, au sud de Sisteron (type Montgervis). Ce silex se collecte facilement sous forme de blocs anguleux rarement corticaux, dans les formations de pente au pied des niveaux calcaires. Les pièces corticales ou semi-corticales sont peu nombreuses $(3,2 \%$ du total du silex bédoulien), témoignant soit d'une mise en forme à l'extérieur du site, soit d'une mise en œuvre rapide des séquences de production comme semble l'indiquer la présence de cortex sur de nombreux supports de plein débitage ;

- silex blond : ont été regroupés sous cette appellation différents types de silex qui représentent $7,4 \%$ du total de la série $(N=999)$ et qui ont en commun une couleur blonde à blond-gris, une texture très fine et une qualité de taille excellente. La plupart de ces types semblent correspondre à de simples variations de faciès et proviennent de galets disponibles dans les alluvions d'âge oligocène situées à l'ouest de la Durance, entre Peyruis et Mirabeau (45 à $80 \mathrm{~km}$ du site). Ces galets sont de dimensions réduites et n'excèdent que rarement $10 \mathrm{~cm}$ dans leur plus grand axe. Comme pour le silex bédoulien, les témoins corticaux ou semi-corticaux ne sont pas très abondants $(5,6 \%)$;

- silex lacustre : c'est quantitativement le troisième type de silex représenté ( $N=536: 3,9 \%$ ). D'une couleur beige-jaune mate et zonée, il contient de nombreux fragments de gastéropodes de dimensions pluri-millimétriques. Ce matériau n'a pas été testé. Toutefois, l'examen des produits indique que ces microfossiles gênent souvent la progression de l'onde de fracture et génèrent des accidents de taille de type rebroussé. Les gîtes se situent dans des niveaux oligocènes proches de Forcalquier (45 à $75 \mathrm{~km}$ de Saint-Antoine). Le silex se présente sous forme de plaquettes de dimensions variables, aisément accessibles, dont le cortex peut être épais. Comme pour les autres variétés, ces zones corticales sont très peu présentes dans la série (3\%);

- silex noir moucheté : C'est le dernier type de silex qui présente une importance quantitative significative $(\mathbf{N}=$ $330: 2,4 \%$ ). La source d'approvisionnement n'est pas connu avec certitude. Des silex macroscopiquement proches sont présent dans les niveaux hauteriviens de Clausonne, à $12 \mathrm{~km}$ au nord-ouest du site, mais des faciès similaires existent dans le haut-Var et dans les Baronnies. En l'absence d'éléments réellement discriminants, même en lame mince, le gîte doit donc être pour le moment considéré comme inconnu.

II s'agit d'une matière première qui semble excellente au vu de la qualité des supports confectionnés. La surface est noire, luisante et piquetée de petits points blancs brillants. L'examen des pièces corticales, peu nombreuses, montre un cortex épais, poreux et indique que ce silex a été collecté sous forme de nodule ;

- divers : quelques types de silex sont présents en très faible quantité $(N=12: 0,1 \%)$. Deux pièces pourraient provenir de quelques kilomètres au sud de Vitrolles (Carixien du Rocher de Hongrie). Quelques autres sont confectionnées dans un silex jaspoïde provenant de la région de Forcalquier. Les autres artefacts sont d'origine inconnue.

L'approvisionnement en silex est donc trés peu varié. Quatre types dominent la série, parmi lesquels le silex bédoulien est très majoritaire puisqu'il en représente les deux-tiers. L'ensemble de l'approvisionnement ne peut donc être considéré comme local. La zone de collecte, pour les variétés qui ont pu être cartographiées, se situe dans le bassin versant de la moyenne vallée de la Durance, peu ou prou entre Sisteron au nord et 


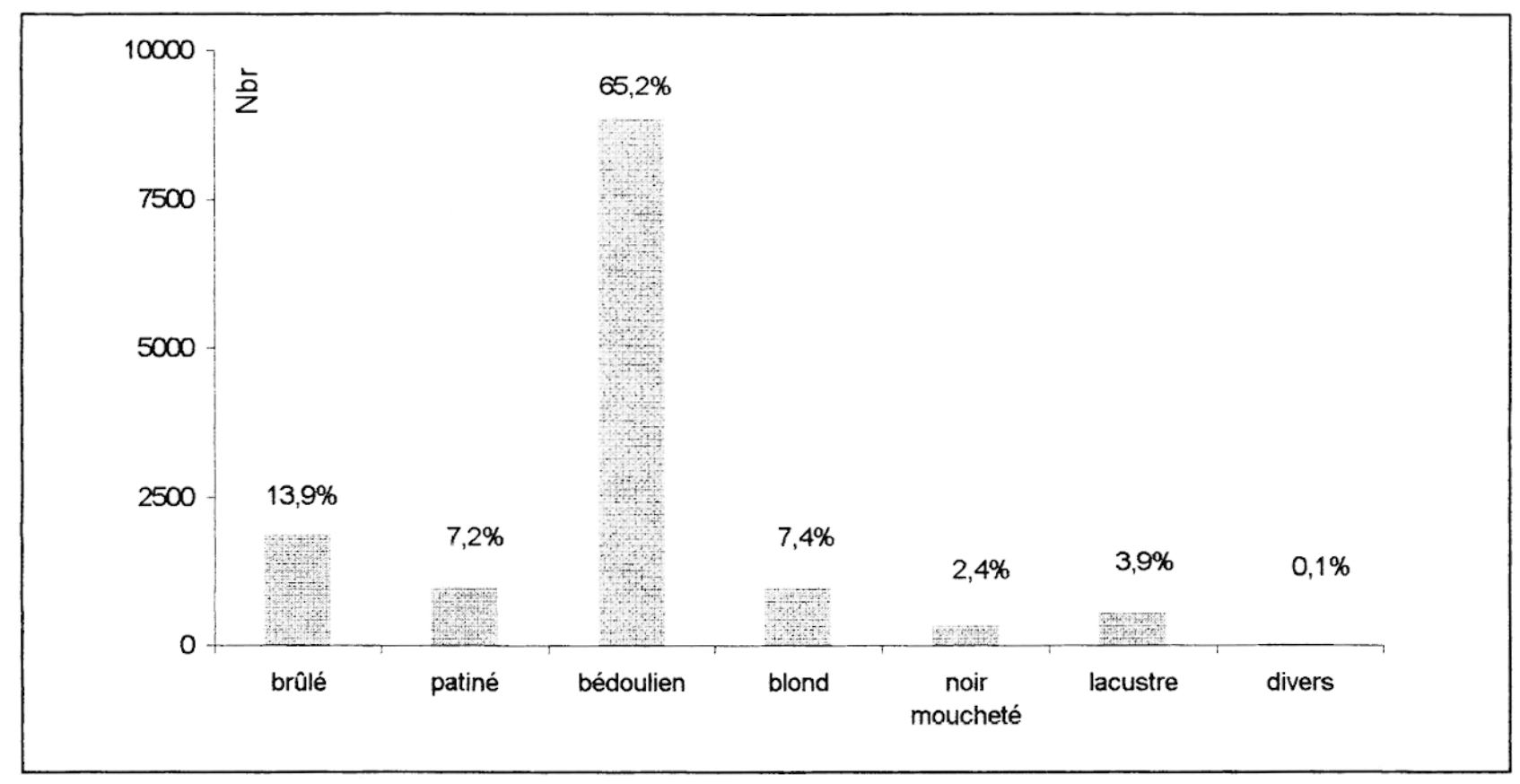

Figure 2 : Saint-Antoine - locus 2.

Décompte des matières premières lithiques $(N=13596)$

(ont été classés comme brûlées ou patinées les pièces dont l'altération empêchait l'attribution à une variété précise de silex ; les pièces brûlées ou patinées mais identifiables ont été classées selon leur matière première)

Lithic raw material counts (burned or patinated artefacts were classed as such only if precise determination of the original raw material is impossible. Identifiable burned or patinated artefacts were sorted according to their raw material)

Manosque au sud. En l'état, il n'a pas été identifié de façon certaine des silex provenant de zones septentrionales à partir du site. La distance minimale aux gîtes est donc d'environ $30 \mathrm{~km}$ vers le sud, sauf pour quelques pièces quantitativement très minoritaires. Cette constance dans la direction de l'approvisionnement suggère fortement une collecte lors de transits du ou des groupes humains d'une zone méridionale encore à définir (Basse Provence, Côte d'Azur...?) vers Saint-Antoine plutôt qu'une acquisition à partir du gisement.

\section{II.4 Le débitage laminaire}

L'important taux d'exploitation des nucléus et le peu de remontages disponibles rendent malaisée l'appréciation des phases initiales du débitage. Toutefois, plusieurs observations peuvent être proposées à partir de l'analyse d'un échantillon de 175 nucléus (fig. 3).

\section{Choix des volumes à débiter:}

Les volumes utilisés comme nucléus sont des blocs, des éclats et des galets (fig. 4).

- l'utilisation de blocs est majoritaire (36 \% des nucléus), en liaison avec la présence importante du silex bédoulien. Ces blocs, de forme tétraédrique le plus souvent, offrent des surfaces d'accueil régulières propices à une mise en forme laminaire. Le module est plus difficile à déterminer. Deux nucléus peu exploités avoisinent 10 $\mathrm{cm}$ dans leur plus grande longueur. Mais les autres sont trop exploités pour pouvoir fournir des indications significatives. Les blocs sur silex lacustre sont proches de plaquettes ;

- les éclats constituent près du quart des supports identifiables $(23,4 \%)$. Une grande partie d'entre eux sont en silex bédoulien. II s'agit surtout de grands éclats corticaux obtenus le long d'un dièdre naturel ; ils possèdent donc une morphologie proche de celle des blocs. Les autres nucléus sur éclat sont surtout sur silex blond pour lequel ils représentent l'essentiel des nucléus. La production de ces éclats est effectuée au percuteur dur dans le grand axe des galets après décalottage d'une extrémité, produisant des éclats de type " couteaux à dos cortical " ;

- l'utilisation directe de galets est rare (3 exemples ; $1,7 \%)$.

La mise en forme : des modalités simplifiées :

Plusieurs éléments permettent de proposer une phase de mise en forme simple et rapide. En premier lieu les produits de cette phase sont peu nombreux. Avec 429 artefacts, le total des éclats corticaux et semi-corticaux dépasse tout juste celui des nucléus. A cette faible représentation répond d'ailleurs la présence de nombreux supports de plein débitage possédant encore des plages corticales. En outre, les lames à crêtes sont très peu nombreuses (28 exemplaires). 

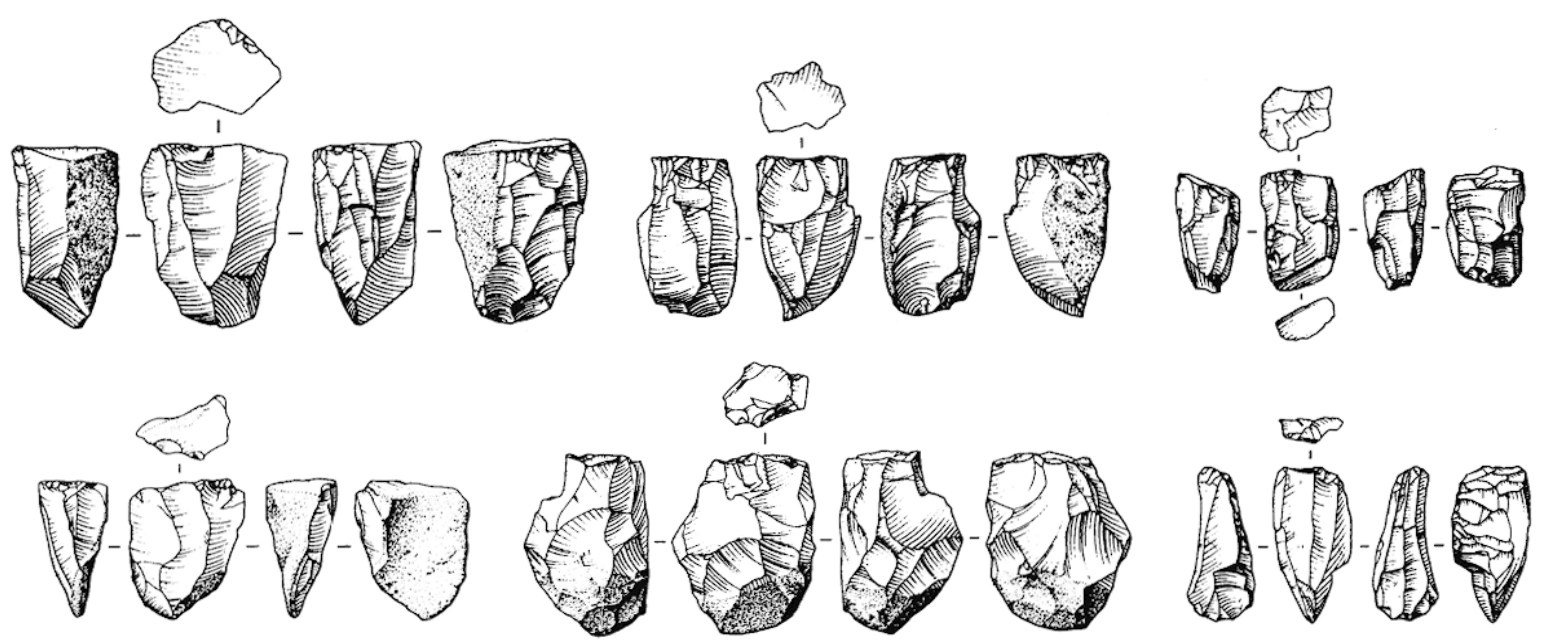

的
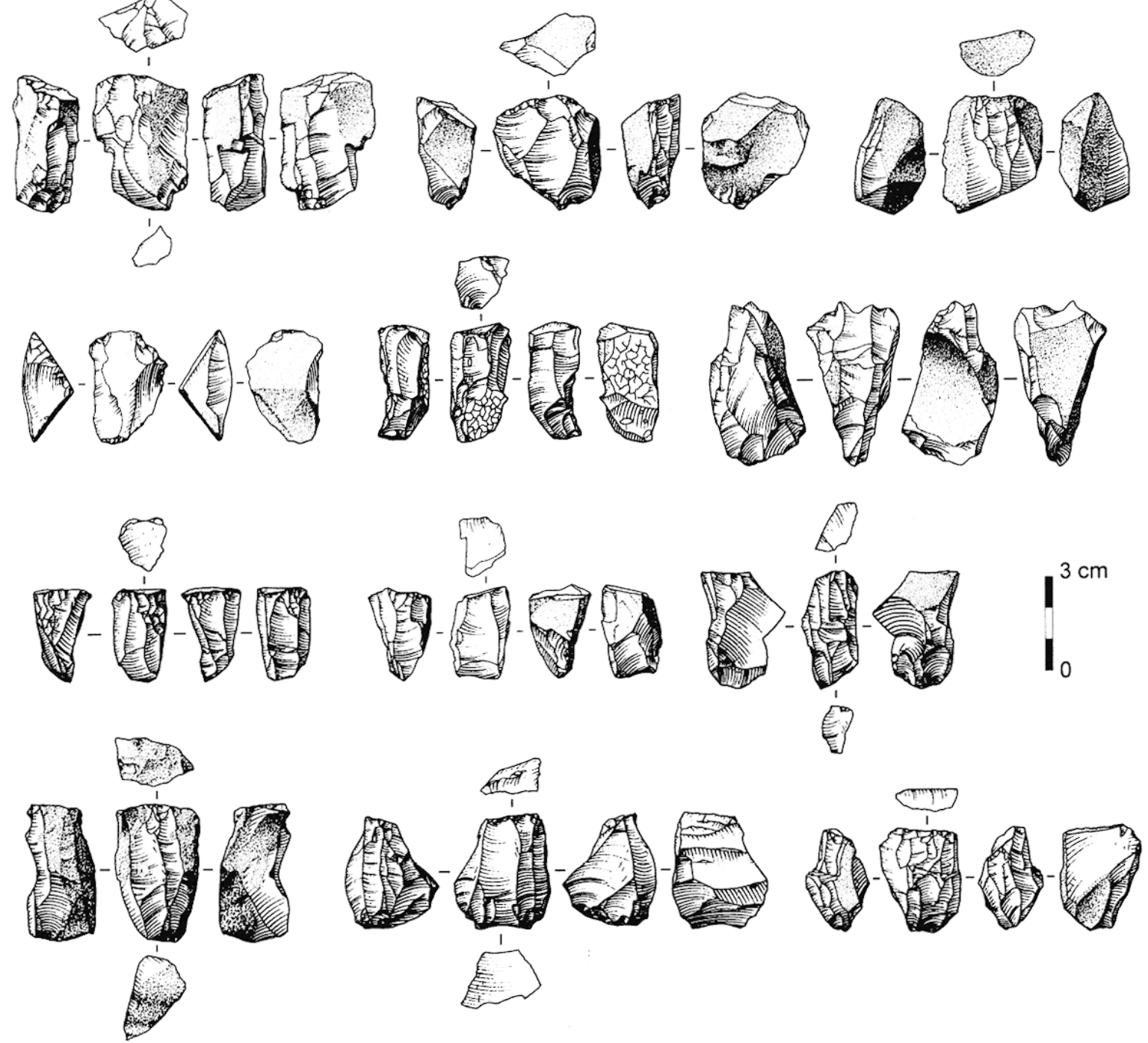

Figure 3 : Saint-Antoine - locus 2.

Nucléus (dessins S. Lancelot, AFAN)

Nucleus 


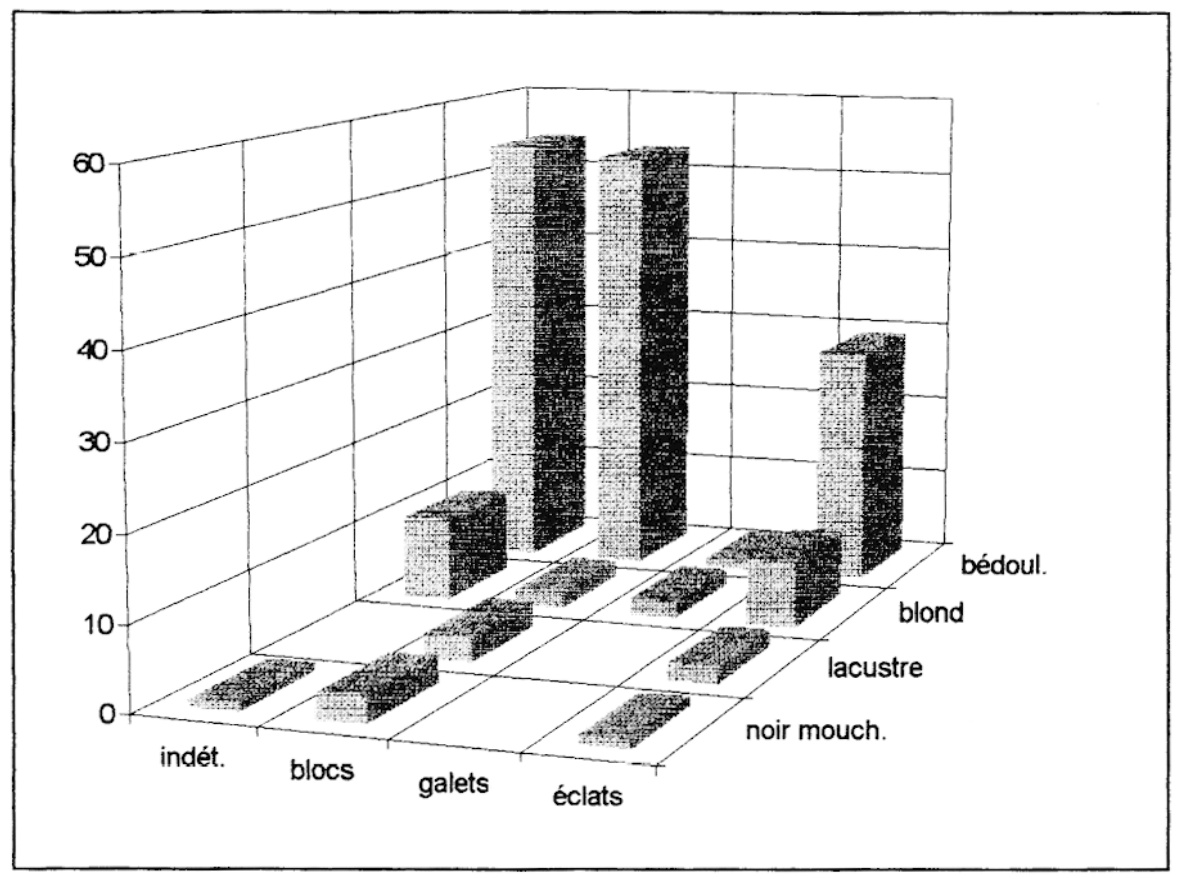

Figure 4 : Saint-Antoine locus 2.

Morphologie initiale des nucléus (échantillon, $\mathrm{N}=$ 175)

Initial morphology of nuclei (sample, $N=175$ )

La distribution des différentes surfaces de travail répond à des principes généraux assez strictement respectés :

- la surface d'initialisation est installée dans la plus grande dimension du nucléus. Sur les nucléus où elle a pu être observée, cette règle ne souffre aucune exception ;

- la largeur de cette surface est inscrite dans la face la plus large en ce qui concerne les blocs. Pour les nucléus sur éclats en revanche, c'est une des deux tranches de l'éclat qui va servir de surface d'initialisation. C'est donc alors la plus faible largeur qui est utilisée ;

- un seul plan de frappe est créé. II ne semble pas qu'une angulation particulière soit recherchée et les plans de frappe peuvent être très obliques ou pratiquement perpendiculaires à la table.

La mise en place des surfaces s'effectue en liaison étroite avec la morphologie des supports et les aménagements sont réduits au strict minimum. Aucun aménagement du dos n'a été relevé et seules quatre crêtes latérales sont présentes. Cette absence peut s'expliquer par la forme initiale des nucléus. Les blocs tétraédriques, comme les éclats exploités sur la tranche, possèdent un cintre naturel propice au débitage. Au bout de cette logique, certains éclats naturellement préformés ont été directement exploités sans aucun aménagement préalable.

Un des aspects les plus caractéristiques de cette phase de mise en forme est la faiblesse des enlèvements destinés à l'établissement de la carène. Les lames à crêtes, partielles ou totales, sont plus orientées vers l'établissement de la rectitude de l'arête que vers sa convexité.
Quelques rares exemplaires, notamment deux nucléus sur galet ovoïde en silex blond, montrent que même pour ces pièces dont la morphologie n'est pas directement exploitable, la mise en forme reste rapide. Elle s'organise à partir de la création du plan de frappe, installé sur une des extrémités de la plus grande longueur. La création de la surface de débitage est alors directement associée à la production d'une lame d'entame corticale, débitée le long du dièdre naturel du galet. Sur un exemplaire toutefois, un des flancs est cintré à partir de la surface ainsi créée.

Bien que rapide, cette mise en forme se révèle efficace à l'examen des produits. Les lames d'entame ou à crête sont en général bien venues et seuls quelques accidents rebroussés ont été relevés, essentiellement sur des entames corticales. Dans ce cas, on observe soit une reprise soignée de la convexité par la création d'une crête totale ou partielle sur la partie non débitée du dièdre, soit un changement immédiat d'orientation et l'extraction, à partir du même plan de frappe, d'une nouvelle lame d'entame sur une autre face du nucléus.

Enfin, ces modalités de mise en forme sont déterminables sur les nucléus laminaires comme lamellaires.

\section{Le plein débitage}

Les toutes premières lames détachées dans cette séquence ne peuvent être distinguées du reste de la série et possèdent déjà les caractéristiques de l'ensemble des supports laminaires. On peut donc considérer que la séquence de plein débitage commence immédiatement après l'extraction de lame d'entame, à crête ou corticale. 


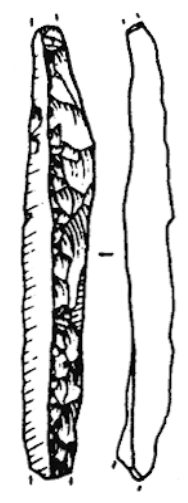

1
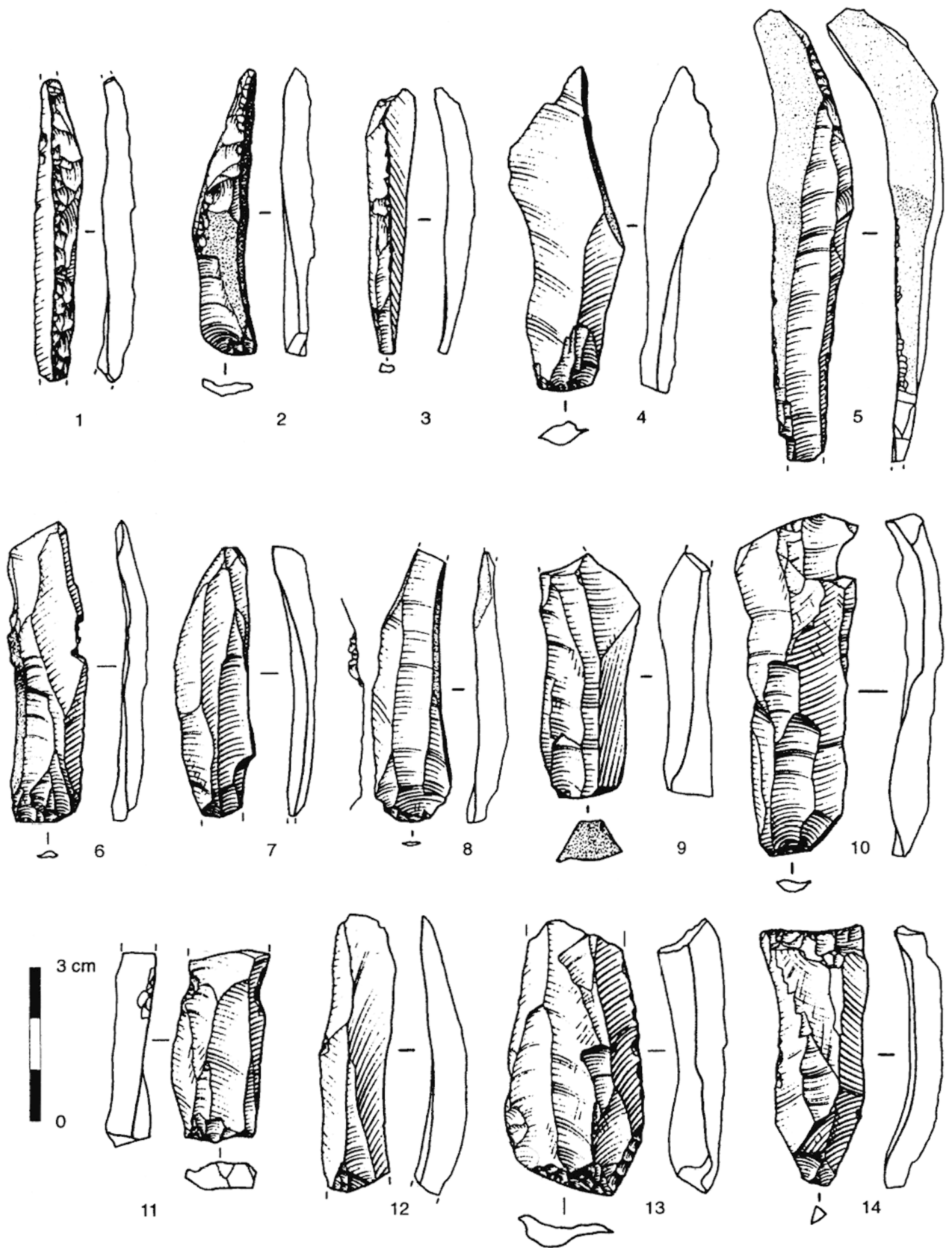

Figure 5 : Saint-Antoine- locus 2, industrie lithique

1-3, 5 : lames demi-crête, 4 : lame de flanc de nucléus, 6-12: lames de plein débitage, 13-14: réfections de table laminaire (dessins $S$. Lancelot, AFAN).

1-3, $5:$ half-crested blades, $4:$ blade obtained on the edge of the nucleus, $6-12:$ blades, $13-14:$ blades used for debitage surface restoration. 
Malgré des différences au niveau des dimensions, l'ensemble de la série possède une "allure " générale homogène. Les supports sont en général plutôt larges, courts avec souvent un très léger rebroussé à l'extrémité distale. Ils sont surtout très rectilignes. L'élément qui montre la plus grande variabilité est sans conteste l'épaisseur (fig. $5: 6-12$ ).

La progression du débitage et le rôle des plans de frappe

\begin{tabular}{lrr}
\hline progression du débitage & fréquence & $\%$ \\
\hline frontale & 71 & 37,8 \\
semi-tournante & 102 & $54,2$. \\
tournante & 15 & 8 \\
\hline
\end{tabular}

Tableau 3 : Saint-Antoine - locus 2

Progression du débitage observée sur les nucléus

Debitage progression on nucleus

Les séries laminaires sont toujours extraites à partir d'un seul plan de frappe préférentiel. Le débitage est en majorité semi-tournant $(54,2 \%$, tableau 3$)$, envahissant progressivement l'un des flancs du nucléus, parfois la totalité de la surface exploitable (15 exemples de débitage tournant). Cette progression sur un flanc semble correspondre à une volonté de contrôle du cintre de la table laminaire. L'ordre des enlèvements joue clairement sur ce phénomène d'auto-entretien, en revenant successivement vers le centre de la table puis ses extrémités afin d'éviter un aplatissement trop rapide de la surface de débitage. Ce choix entraîne la production de nombreux supports de plein débitage partiellement corticaux, parfois repris en demi-crête, qui ne sont donc en aucune façon liés aux premières phases du débitage (fig. $5: 1$ 5). En même temps, ce balancement entre centre et bords de la surface de débitage produit des supports morphologiquement différenciés : plutôt larges et à section trapézoïdale aplatie pour les lames issues du centre, plutôt étroits, épais et à section triangulaire pour les lames issues des bords.

Le plan de frappe a ainsi un rôle central dans la gestion du volume. Ce rôle est encore renforcé par son utilisation pour le contrôle de l'angle plan de débitage/plan de frappe et dans les phases de réfection de la table laminaire :

- le maintien de l'angulation est un des problèmes constants sur ces nucléus. La faible carène initiale des surfaces de débitage entraîne leur aplatissement rapide. Deux solutions semblent être utilisées. Quand le nucléus est encore très productif $(L>5 \mathrm{~cm})$, la réfection de l'angle est obtenue par l'enlèvement d'une tablette de ravivage. Ces tablettes, représentées par 178 exemplaires dans la série, sont en effet pratiquement toujours de grande dimension par rapport à la taille d'abandon des nucléus. Si les nucléus sont de petite taille, la production est arrêtée sur cette surface ;

- la faible convexité des tables laminaires occasionne des enlèvements rebroussés relativement abondants qui nécessitent des phases de réfections des tables laminaires. Ces réfections sont obtenues par la production de grands éclats au percuteur dur à partir du plan de frappe. Ces éclats éliminent en règle générale une grande partie de la surface de la table laminaire (fig. 5 : $13 \& 14$; fig. $6: 1,2 \& 4$ ). Très efficace, cette technique est néanmoins très consommatrice en matière première ;

- Enfin, c'est toujours à partir du plan de frappe que s'effectue le contrôle de la rectitude des nervures-guides par l'enlèvement de produits lamellaires courts dont l'objectif est de recentrer les nervures par rapport à l'axe de débitage du nucléus.

Plus d'un-tiers des nucléus est exploité selon une progression frontale (tableau 3). II s'agit pour l'essentiel de nucléus sur éclat pour lesquels la phase initiale du débitage s'effectue sur la tranche. Le très fort cintre naturel que possède alors le nucléus rend inutile l'auto-entretien que génèrent les débitages semi-tournants. Les séquences d'entretien semblent en revanche identiques quelque soit le mode de progression du débitage et les réfections sont essentiellement effectuées à partir du plan de frappe.

Le premier plan de frappe est conservé jusqu'à l'abandon ou l'épuisement du nucléus ou bien jusqu'à ce que la morphologie du nucléus ne permette plus l'entretien de la table et du plan de frappe et donc la poursuite du débitage. Plusieurs modalités sont alors possibles :

- la même face est exploitée à partir d'un plan de frappe opposé. C'est la modalité la plus usuelle (71 cas sur 162 (11) soit $44 \%$ ). On observe souvent un léger décentrage du débitage par rapport au premier plan de frappe, avec l'envahissement progressif du flanc opposé à celui utilisé lors de la production de la série précédente. Ce décentrage permet de redonner du cintre à la table laminaire ;

- une nouvelle face est exploitée à partir du même plan de frappe. Cette face peut être un des flancs ou le dos du nucléus. Cette opération s'accompagne souvent de réaménagements du volume sous forme de création d'une néocrête et d'enlèvements perpendiculaires à la table destinés au cintrage ;

- un nouveau plan de frappe est ouvert en liaison avec l'exploitation d'une nouvelle surface. Dans ce cas, les modalités sont les mêmes que pour la création de la première table et les aménagements sont là aussi réduits au minimum. 

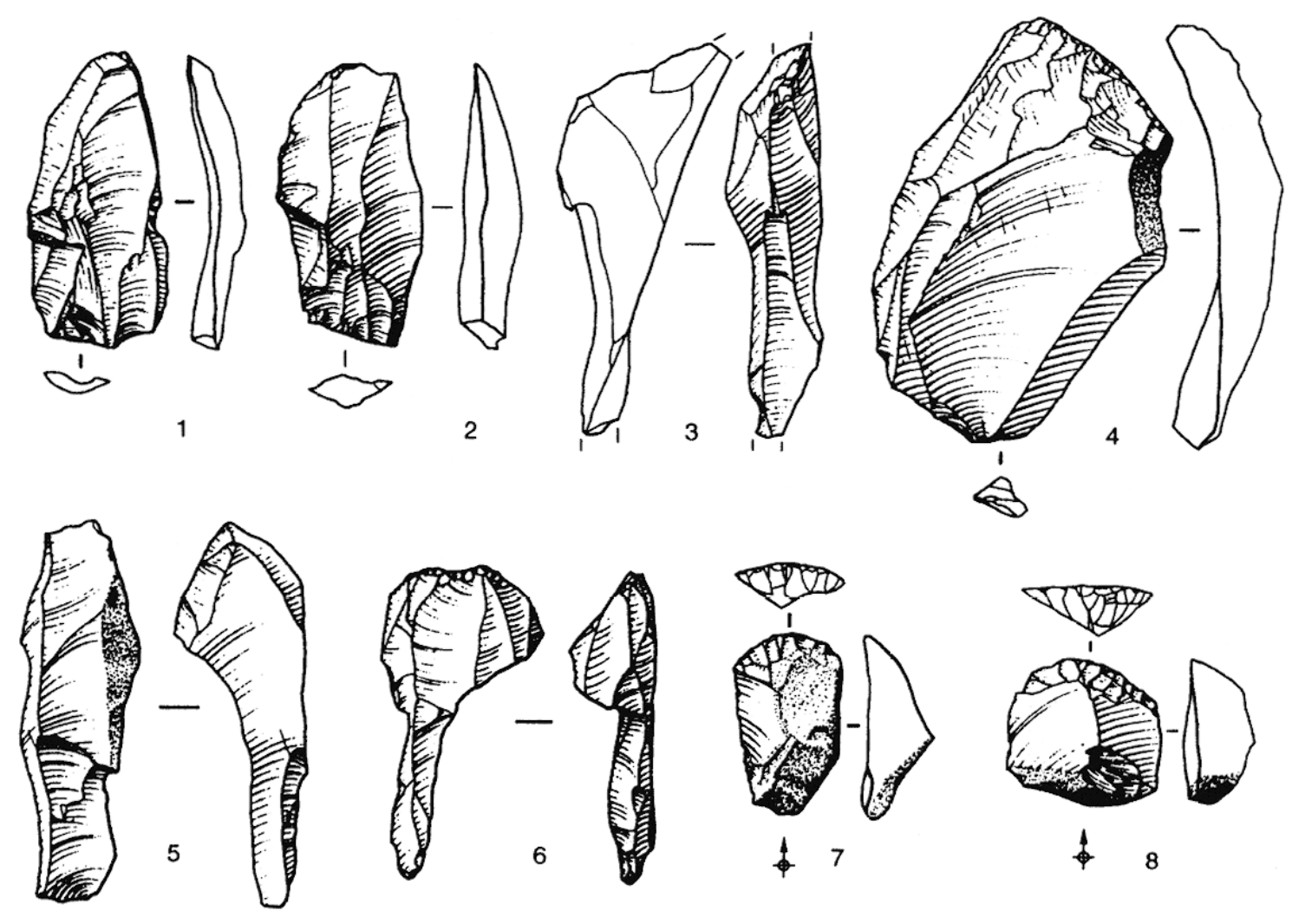

\section{Sertoren}
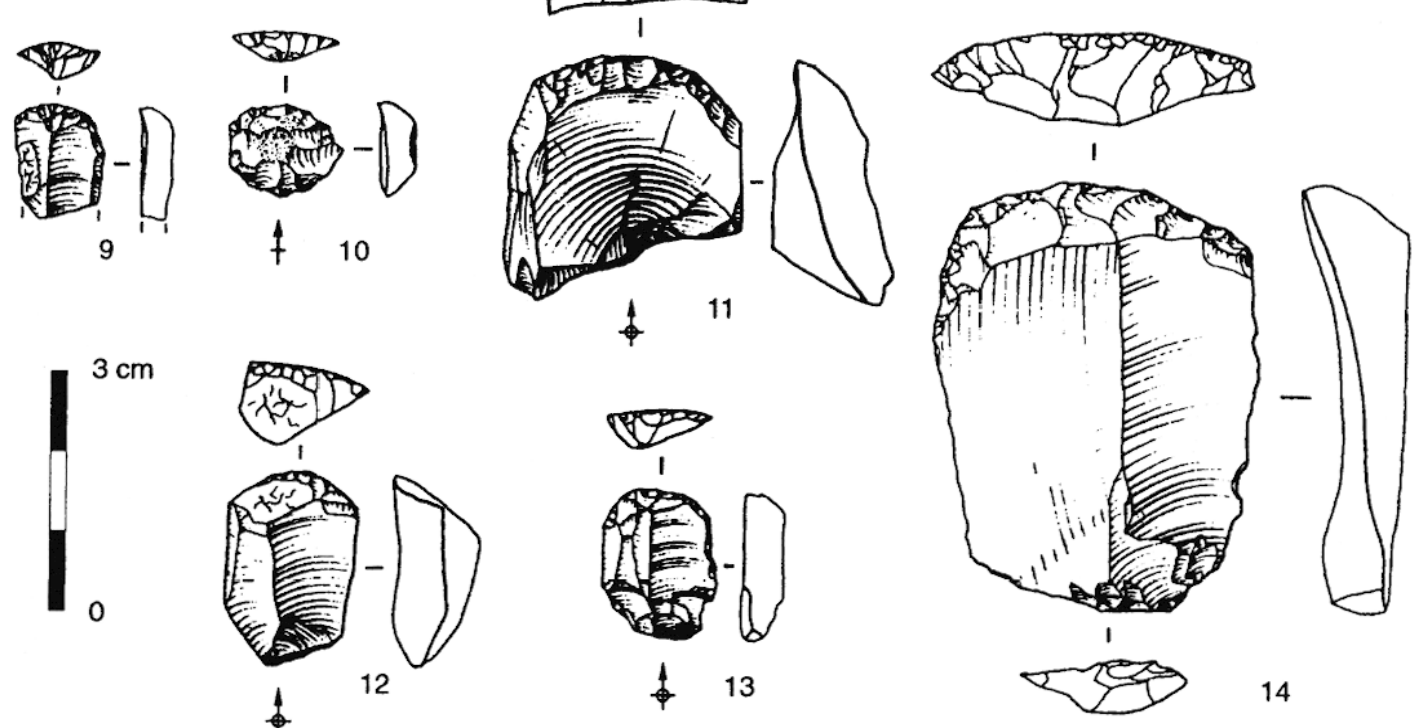

Figure 6 : Saint-Antoine - locus 2 , industrie lithique

$1,2,4$ : réfections de table laminaire, 3 : lame d'initialisation d'une nouvelle surface laminaire, 5-6 : lamelles outrepassées, 7-14: grattoirs (dessins S. Lancelot, AFAN).

1, 2, 4 : débitage surface restoration, 3 : blade used to begin a new debitage surface, 5-6: plunging bladelets, 7-14: scrapers 
Quelques soient les modalités, l'installation des surfaces de débitage recherche toujours le plus grand axe d'allongement du nucléus. C'est probablement pour cette raison que les débitages croisés sont pratiquement inexistants ( 1 seul exemple).

\section{Techniques de détachement}

L'observation de plusieurs caractères : la rectitude importante des produits, le taux d'esquillement des talons et des caractères secondaires teis que la fréquente présence d'esquilles bulbaires et de cônes incipients... nous ont conduit à soupçonner l'utilisation éventuelle d'un percuteur de pierre tendre (Gagnepain \& Bracco 1996 : 21). L'examen d'une fraction importante de la série par J. Pelegrin (URA 28, Meudon) - que nous tenons à remercier chaleureusement -, a permis de confirmer ce diagnostic. Les commentaires du paragraphe ci-dessous sont pour l'essentiel issus de ses observations.

En ce qui concerne la phase de plein débitage, les critères suivants peuvent être décrits :

\begin{tabular}{lrr}
\hline types de talon & fréquence & $\%$ \\
\hline cortical & 5 & 1,5 \\
linéaire & 7 & 2 \\
lisse & 95 & 27,1 \\
lisse abrasé & 173 & 49,4 \\
facetté & 7 & 2 \\
esquillé & 63 & 18 \\
Total & 350 & \\
\hline
\end{tabular}

Tableau 4 : Saint-Antoine - locus 2

Décompte des talons des lames et lamelles brutes (échantillon, $\mathrm{N}=350$ )

Blades and bladelets butts (sample)

- la préparation des talons concerne un peu plus de la moitié d'entre eux $(51,4 \%$ de l'ensemble de l'échantillon et $62,7 \%$ des talons conservés, tableau 4). Cette préparation s'effectue par abrasion du plan de frappe vers la surface de débitage, la zone d'impact proprement dite restant lisse. Cette abrasion est souvent importante et le recul de la corniche prononcé. Son rôle est de renforcer le point d'impact ;

- l'esquillement des talons concernent près d'un cinquième des pièces. Dans ce cas, on observe un éclatement, partiel ou total, du talon à partir d'une onde de fracture se développant depuis le point d'impact ;

- les esquilles bulbaires sont très abondantes. Elles n'ont pas été quantifiées, mais leur présence est attestée sur beaucoup plus de la moitié des lames et lamelles. Elles sont en général assez étroites, de direction oblique par rapport à l'axe de débitage et leur extrémité proximale vient toucher le talon. Sur quelques exemplaires, l'esquillement est très important et emporte une partie de la face inférieure du support ;

- la rectitude de nombreux produits a déjà été relevée. Elle est associée à un angle d'éclatement souvent très ouvert et proche de $90^{\circ}$.

II reste à souligner l'importante présence des talons lisses, non préparés, qui constituent un peu plus du quart de la série $(27,1 \%)$. La mise en relation de ces talons avec la matière première (fig. 7) montrent que s'ils sont surtout abondants dans le silex bédoulien, ils constituent la moitié des talons des lames en silex lacustre, silex dans lequel ce sont surtout des lames, et non des lamelles, qui ont été obtenues. C'est d'ailleurs pour ce seul silex que les talons lisses abrasés ne sont pas proportionnellement majoritaires. L'examen des supports suggère que l'absence de préparation des talons est associée à une percussion très à l'intérieur du plan de frappe, générant des talons épais. Le risque de "casser" au débitage est alors très faible et il n'y a pas nécessité de renforcer le point d'impact par une abrasion. Les talons lisses sont donc en général associés aux productions de plus grande taille.

\section{Données morphométriques sur les supports laminaires}

Les dimensions générales des supports laminaires sont réduites. Pour l'échantillon étudié, la longueur est comprise entre 13 et $85 \mathrm{~mm}$ et la largeur entre 4 et $33 \mathrm{~mm}$. L'examen des classes de longueur des lames et lamelles brutes indique clairement que les artefacts entre 18 et 55 $\mathrm{mm}$ de longueur sont très majoritaires (fig. 8). La quasiabsence de pièces inférieures à $18 \mathrm{~mm}(\mathrm{~N}=8)$ suggère que le débitage est volontairement stoppé à cette limite. Bien que peu abondantes, les pièces supérieures à 55 $\mathrm{mm}$ sont mieux représentées.

Comme pour les longueurs, les largeurs des supports laminaires sont peu variées, essentiellement comprises entre 6 et $17 \mathrm{~mm}$ (fig. 9). Les même remarques concernant les dimensions en-deçà et au-delà de ces limites sont possibles. Au moins pour ces deux paramètres, les objectifs du débitage semblent peu diversifiés et correspondent à un module général bien individualisé.

\section{La phase finale du débitage et l'abandon des nucléus}

S'il est difficile de quantifier le taux d'exploitation des nucléus (absence de remontages), il est cependant clair que la production est souvent importante. En témoignent notamment les nombreux nucléus qui montrent l'exploitation successive de plusieurs tables.

Les dimensions d'abandon des nucléus (dimensions de la totalité des tables laminaires entières, il peut y en avoir plusieurs par nucléus) sont remarquablement stables (fig. 10), inférieures ou égales à $5 \mathrm{~cm}$ pour les longueurs (la plus grande variabilité des largeurs correspond au mode d'exploitation, semi-tournant ou frontal). Seuls deux nucléus ont été abandonnés avant cette limite. 


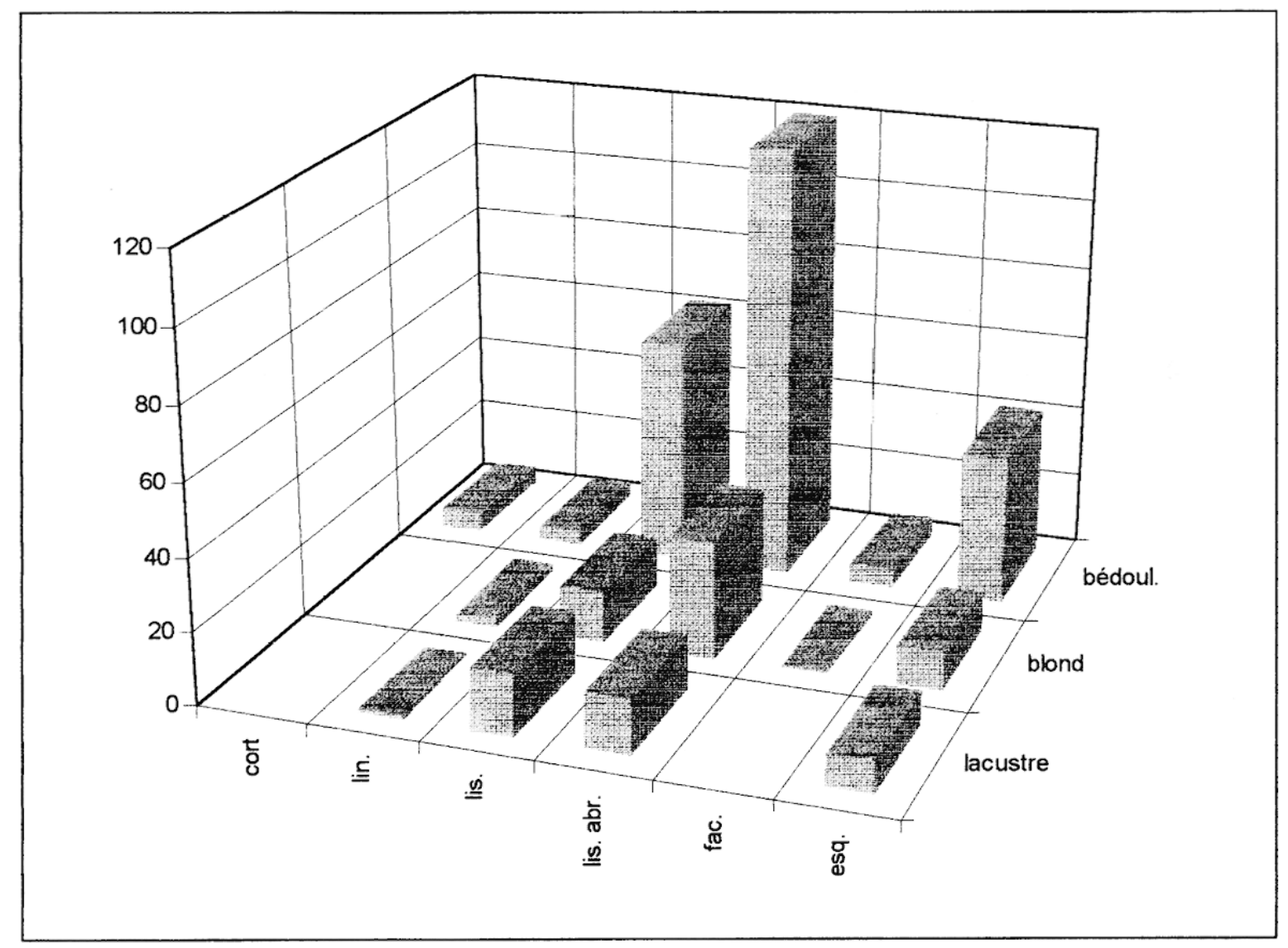

Figure 7 : Saint-Antoine - locus 2

Décompte des talons sur les lames et lamelles brutes par matière première (échantillon, $N=339$ )

Counts of blade and bladelet butts by raw material (sample)

Enfin, juste avant l'arrêt de la production, la tentative d'enlèvement d'un dernier support sur des tables souvent extrêmement plates a plusieurs fois produit une lamelle très large, parfois un véritable éclat, finissant de rendre inutilisable le plan de débitage qui peut même se retrouver concave. Ces supports pourraient témoigner d'une chute de soin à la fin de la séquence de production.

\section{II.5 Le débitage d'éclats}

Il est très peu documenté. Son étude demanderait pour être complète l'analyse approfondie de l'ensemble des éclats afin de déterminer ceux issus d'un débitage laminaire (mise en forme, entretien...) et ceux qui représentent réellement des supports de première intention. Ce travail n'a pas encore été réalisé.

Les deux nucléus sont les seules pièces immédiatement informatives. II s'agit dans les deux cas d'un débitage discoïde sur galet. Un seule face est exploitée à partir d'une surface réserve dont l'angulation est soigneusement entretenue par des enlèvements qui partent de la surface de débitage. Les éclats, par définition de direction centripète, sont envahissants et peu épais. L'examen des points d'impacts indique une percussion au percuteur dur. Autant que l'on puisse en juger, la productivité semble importante. En effet, plusieurs générations d'enlèvements de contrôle de l'angulation sont décelables sur la face réserve.

\section{II.6 Quelques observations sur le débitage laminaire de SAV - locus 2}

A ce stade peuvent être proposées quelques premières observations sur les modalités de débitage :

- introduction de la matière première : la faible représentation des témoins corticaux peut être mise en relation avec le faible investissement décelé pour la mise en forme des nucléus et l'importance des nucléus sur éclats. Elle ne présume donc pas d'une première opération de décorticage en dehors du site. Cette hypothèse ne pourra toutefois être définitivement établie que par des remontages abondants ; 


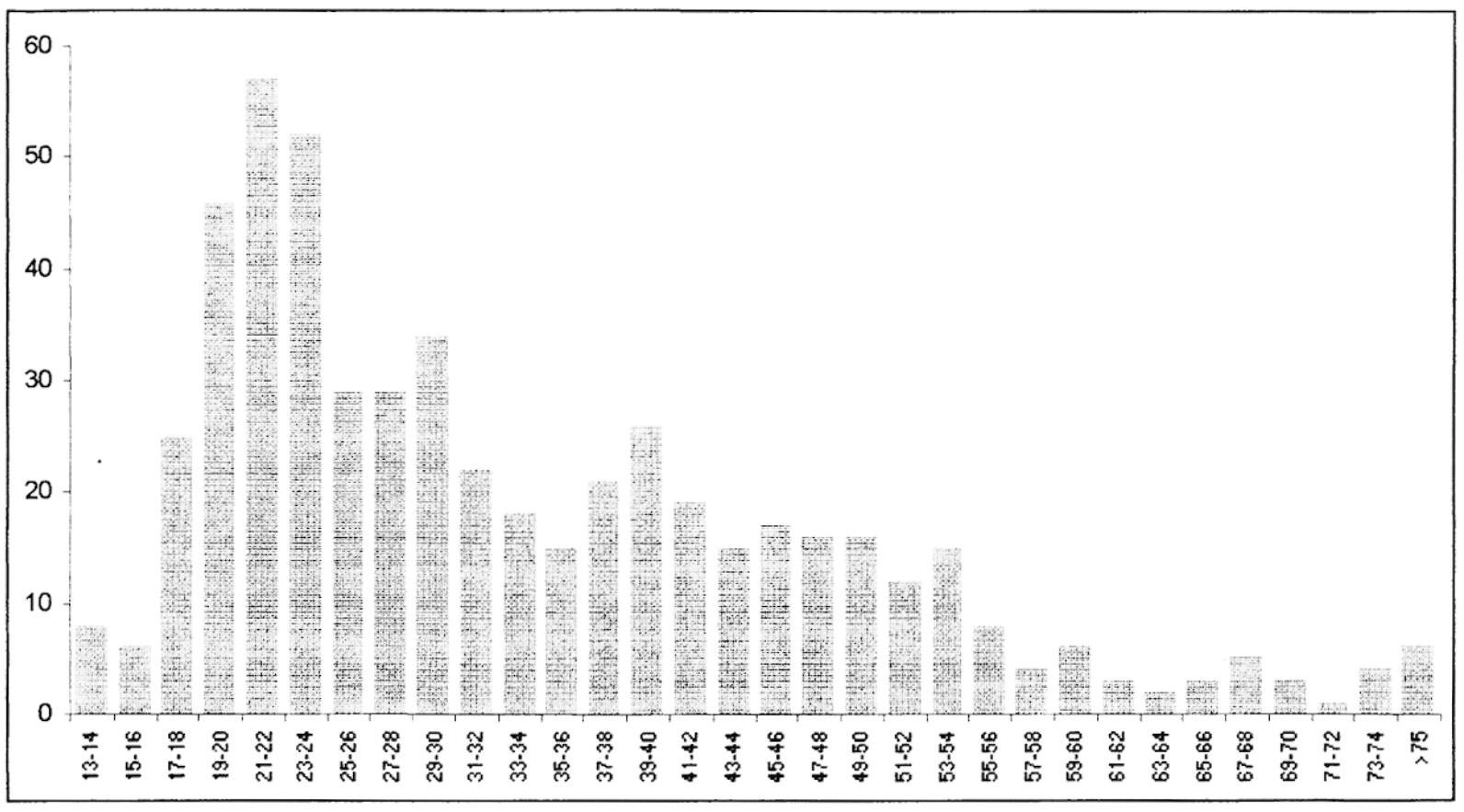

Figure 8 : Saint- Antoine - locus 2

Longueur des supports laminaires entiers (lames et lamelles brutes + armatures, échantillon, $\mathrm{N}=544$, classes de $2 \mathrm{~mm}$ ) Blade and bladelet lengths (unretouched blades and bladelets + projectile points, sample, $N=544$ )

- représentation des différents témoins de plein débitage : il existe une distorsion quantitative entre les sousproduits du débitage des lames et les lames retrouvées $(>5 \mathrm{~cm})$. Cette distorsion est particulièrement visible au niveau des tablettes de ravivage. La majorité des 178 tablettes peut être attribuée à une phase de production de lames. Les tablettes issues d'un débitage lamellaire sont très rares. Ce nombre de 178 n'est pas en correspondance avec la très faible représentation des lames de plein débitage. En outre, ces dernières sont très souvent semi-corticales. Beaucoup d'entre elles sont des lames de flancs, qui participent à l'élargissement de la table laminaire dans le cadre d'un débitage semitournant. Les lames entièrement décortiquées sont peu nombreuses. Or, l'examen des tablettes montre clairement qu'elles ont existé. Signalons aussi que les lames de longueur supérieure à $5 \mathrm{~cm}$ étant peu nombreuses, nous avons tenté de les remonter systématiquement mais sans résultats. Le seul remontage réalisé sur ces grandes pièces concerne une tablette de ravivage sur un éclat de décorticage, c'est-à-dire un remontage de sous-produits de la chaîne opératoire. II est alors possible de proposer un prélèvement de ces lames sur le stock industriel au moment du départ des groupes humains. En l'état, cette seule hypothèse nous semble capable de rendre compte de la distorsion numérique entre les sous-produits et les supports de plein débitage ainsi que de l'absence de remontages ;

- économie des matières premières : Les modalités de débitages mis en œuvre sont parfaitement efficaces.
Toutefois, elles sont surprenantes dans ce contexte d'approvisionnement allochtone. Les phases de réfection des tables laminaires par exemple, par grands enlèvements envahissants sur la table à partir du plan de frappe, sont extrêmement dispendieuses en matière première. D'autres solutions, comme la confection de crêtes latérales dès la mise en forme du nucléus, auraient pu être employées.

En réalité, l'examen de l'ensemble de la chaîne opératoire démontre que l'on ne se situe absolument pas là dans un contexte de pénurie de la matière première, malgré l'absence presque total de silex dans un rayon de $30 \mathrm{~km}$. Cette observation exprime bien la complexité des comportements des groupes humains de la fin du Pléistocène et des réponses non linéaires (dans ce cas précis en fonction de la distance au gîte) qui ont pu être apportées à la diversité des situations.

\section{II.7 La transformation des supports}

Le taux de transformation des supports est relativement important : 2671 pièces sur 13293 supports potentiels (ensemble des pièces moins les nucléus) ont été retouchées, soit $20 \%$.

Sélection des supports pour la confection des outils retouchés

Pour l'ensemble de l'outillage, l'utilisation de lames et de lamelles est prépondérante $(95,3 \%$; tableau 5), en accord avec l'objectif principal du débitage. 


\begin{tabular}{lrr}
\hline & fréquence & $\%$ \\
\hline éclats & 102 & 3,8 \\
lames/lamelles & 2544 & 95,3 \\
indéterminés & 25 & 0,9 \\
Total & $\mathbf{2 6 7 1}$ & \\
\hline
\end{tabular}

Tableau 5 : Saint-Antoine - locus 2

Proportion éclats - supports laminaires pour la confection des outils retouchés

Flake / blade-bladelet ratio for retouched tools confection

L'importance numérique des armatures à dos, uniquement fabriquées sur lamelles, "écrase " en partie la vision de la sélection des supports pour l'ensemble de la série retouchée. C'est pourquoi un décompte a été effectué sans ces pièces (tableau 6). On constate alors que l'utilisation des éclats est importante pour la confection des outils retouchés hors armatures à dos. Au total donc, quatre groupes peuvent être distingués :

\begin{tabular}{lrrr}
\hline & éclats & lames & indét. \\
\hline burins & 1 & 4 & 0 \\
grattoirs & 90 & 99 & 10 \\
pièces esquillées & 9 & 1 & 2 \\
pièces à encoches & 0 & 7 & 2 \\
pièces tronquées & 0 & 7 & 0 \\
pièces à ret. abruptes & 0 & 6 & 11 \\
pièces à ret. discontinues & 2 & 7 & 0 \\
Total & 102 & 131 & $\mathbf{2 5}$ \\
& $(39,5 \%)$ & $(50,8 \%)$ & $(9,7 \%)$ \\
\hline
\end{tabular}

Tableau 6 : Saint-Antoine - locus 2

Proportion éclats/supports laminaires pour la confection des outils retouchés hors armatures

Flake / blade-bladelet ratio for retouched tools confection without backed artefacts

- des outils majoritairement confectionnés sur lames: les burins, pièces à encoches, pièces tronquées, pièces à retouches abruptes et pièces à retouches discontinues font partie de ce groupe. L'ensemble des supports laminaires présente des caractéristiques communes. II s'agit presque uniquement de lames (deux lamelles uniquement pour les pièces à encoches). En général, elles sont peu régulières, très souvent partiellement corticales (lames de bord de nucléus) ;

- des outils majoritairement confectionnés sur éclats : les pièces esquillées en sont les seules représentantes. II est assez difficile, en raison de l'importante transformation liée à la retouche, de déterminer de quelles catégories d'éclats il s'agit. Les morphologies sont en tout cas très variables et les pièces fines et allongées coexistent avec des éclats épais et courts ;
- des outils sur supports variés : ce sont uniquement les grattoirs (fig. $6: 7-14$ ). Une très grande partie des éclats identifiables peut être assimilé à des sous-produits du débitage laminaire. Deux catégories ont été particulièrement utilisées : les tablettes de ravivage et les éclats d'entretien de la table laminaire. Ces pièces possèdent une forme générale robuste et des bords semi-abrupts à abrupts propices à la confection des fronts de grattoirs On note aussi la présence plus sporadique d'éclats semi-corticaux correspondant soit à la phase de mise en forme des nucléus, soit à des séquences d'entretien (cintrage des flancs). Les lames correspondent le plus souvent à des produits de bord de table, partiellement corticaux. Comme pour les éclats, les volumes sont très variés sauf au niveau de l'épaisseur ;

- des outils uniquement confectionnés sur lamelles : c'est le groupe des armatures à dos.

\section{Choix des supports d'armatures}

L'utilisation de lamelles est semble exclusive pour la confection des armatures à dos. Un seul contre-exemple a été relevé pour une pièce façonnée sur une extrémité distale d'éclat. L'ensemble des supports sélectionnés est très rectiligne mais c'est une constante dans le débitage de Saint-Antoine - locus 2 et cette constatation ne peut avoir valeur de choix.

Ce groupe des armatures à dos peut être divisé, dans un premier temps, en trois classes : les pointes, les lamelles et les pièces à crans (fig. 11). Beaucoup de pièces, fragmentaires, restent indéterminées et seules les pièces entières et les extrémités apicales ont pu permettre une discrimination entre pointes et lamelles. Sur cette base, les pointes représentent environ les deux-tiers des armatures. Les pièces à crans sont extrêmement rares.

La comparaison des matières premières entre l'ensemble de l'industrie et les armatures met en évidence un prélèvement sélectif (fig. 12). Le silex blond représente $42,9 \%$ du silex utilisé pour les armatures contre $7,3 \%$ pour le total de l'industrie. A un degré moindre, le silex noir moucheté est lui aussi sur-représenté dans les armatures. A l'inverse, le silex lacustre est sous-représenté par rapport au total de la série. Les pièces en bédoulien restent majoritaires mais leur représentation est de $20 \%$ inférieure au total de l'industrie.

\section{Données morphométriques}

La longueur des armatures à dos est relativement peu normée et varie entre 13 et $42 \mathrm{~mm}$, la majorité d'entre elles se situant entre 18 et $35 \mathrm{~mm}$ (fig. 13). On constate une taille minimale et moyenne des pointes supérieure à celle des lamelles.

La largeur des pièces après retouche apparaît au contraire comme extrêmement bien calibrée. Les troisquarts des pièces ont une largeur de 4 à $5 \mathrm{~mm}$, moins de $8 \%$ sont plus larges (fig. 14). 


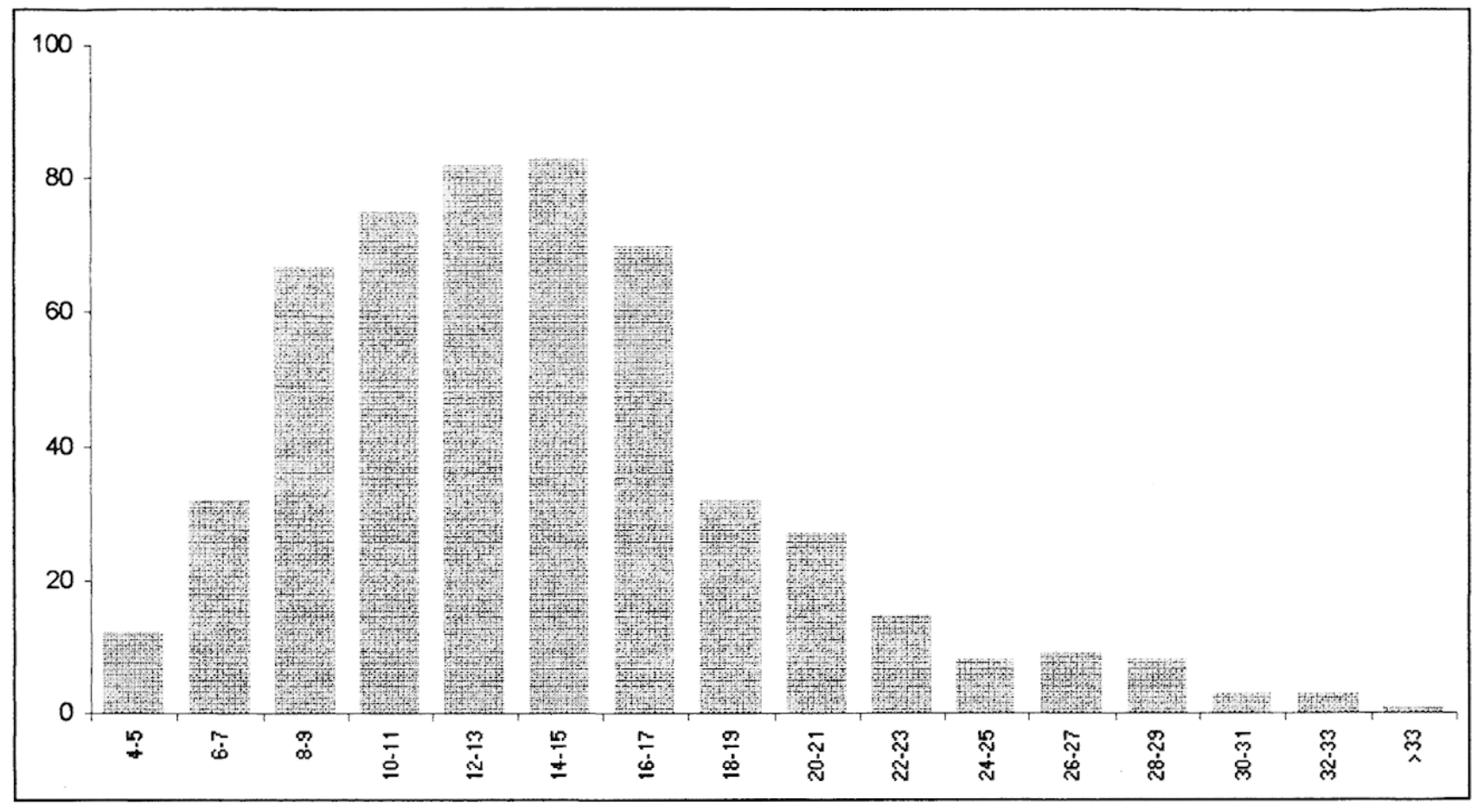

Figure 9 : Saint- Antoine - locus 2

Largeur des lames et lamelles brutes (échantillon, $N=527$, classes de $2 \mathrm{~mm}$ )

Blade and bladelet widths (sample, $N=527$ )

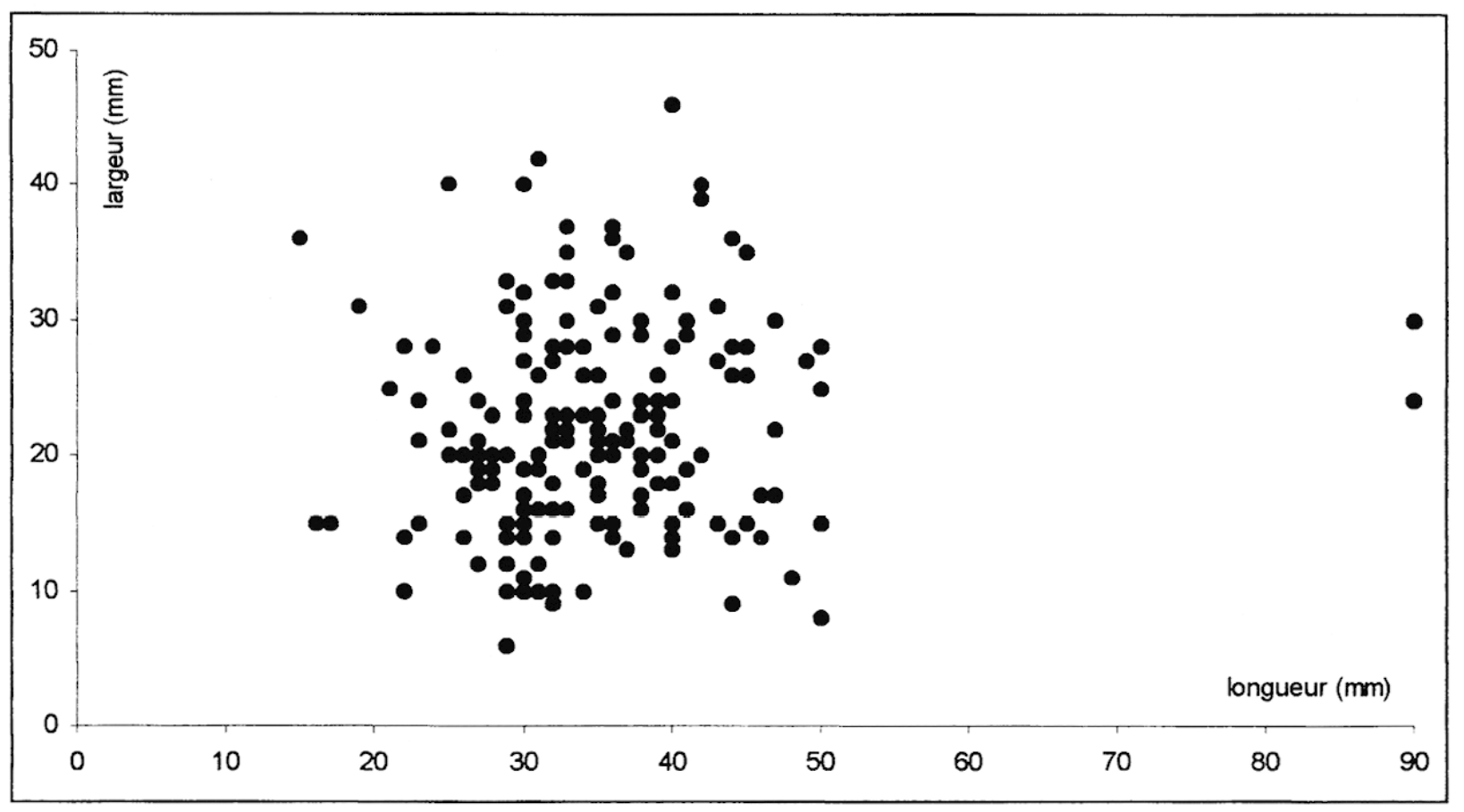

Figure 10 : Saint- Antoine - locus 2

Rapport longueur sur largeur des tables laminaires à l'abandon des nucléus (échantillon, $N=173$ )

Length/width ratio of the debitage surface (sample, $N=173$ ) 


\section{Données qualitatives}

Bien que l'étude des armatures soit encore incomplète ${ }^{(12)}$, quelques observations peuvent être proposées.

La section des lamelles sélectionnées pour la confection des armatures est soit triangulaire, soit trapézoïdale. II semble que les sections triangulaires soient principalement associées aux pointes à dos. Cette morphologie provient des supports débités en bord de table. La hauteur des dos n'a pas été mesurée mais l'impression générale est qu'elle est plus importante pour les pointes que pour les lamelles et pourrait expliquer cette préférence pour des supports triangulaires. En outre, la progression de l'onde de fracture selon une seule nervureguide pour les lamelles de bord de table produit des pièces naturellement pointues, facilitant ainsi la mise en place de la forme définitive des pointes.

Une interprétation complémentaire peut aussi être avancée. La fonction classiquement présumée des pointes en armatures axiales perçantes nécessite éventuellement une plus grande robustesse que l'insertion des lamelles en armatures latérales. Mais en l'absence de véritables données permettant d'assigner à chacun des types d'armatures une fonction et une position assurée dans la composition d'une pièce composite, cette hypothèse ne peut pour l'instant dépasser le domaine de la proposition.

\section{II.8 Analyse typologique}

- les burins : sur les cinq exemplaires, quatre sont d'angle et un dièdre. Des réaffûtages sont présents sur quatre pièces ;

- grattoirs : ils sont extrêmement variés. L'une des rares caractéristiques communes est l'absence de grattoirs doubles. Sur lames, le front est toujours situé en bout. II est semi-abrupt, convexe et certaines pièces semblent avoir été plusieurs fois réaffûtées. Sur éclat, la localisation du front est plus variable. Les bords les plus longs semblent être souvent utilisés mais des fronts courts sont aussi présents. Quelques pièces sont très petites, à la limite de la préhension, et possèdent une retouche qui fait tout le tour de l'objet (fig. $6: 10$ ). Nous les avons classés dans les grattoirs "faute de mieux " mais une analyse tracéologique serait à envisager pour déterminer la fonction de ces artefacts ;

- pièces esquillées : mis à part un exemple, les pièces esquillées sont toutes bifaciales et les esquillements partent de deux bords opposés. Ces pièces peuvent être rattachées au deuxième stade d'exhaustion défini par Tixier (1963 p. 147). Il n'a pas été relevé de "bâtonnets" (stade 3 de Tixier), mais il faudrait réexaminer l'ensemble de la série et notamment les pièces issues du tamisage pour s'en assurer ;
- pièces à encoches : deux groupes peuvent être proposés. Trois pièces ont une seule encoche façonnée par plusieurs petites retouches semi-abruptes. Les quatre autres pièces montrent une succession d'encoches clactoniennes continues. Ces pièces sont à la limite de denticulés ;

- pièces tronquées : elles constituent l'ensemble le plus homogène des outils retouchés (hors armatures). Mis à part une troncature rectiligne, toutes les autres pièces, sur lame uniquement, présentent une troncature concave distale normale abrupte ;

- pièces à retouches abruptes : il est extrêmement difficile de décrire ces pièces qui sont toutes fragmentaires. Signalons que la retouche est toujours partielle, profonde, probablement effectuée par pression ;

- pièces à retouches discontinues : il s'agit essentiellement de pièces portants des encoches discontinues dont certaines peuvent être des témoins d'utilisation ou des retouches mécaniques ;

- les armatures à dos : Leur analyse est gênée par le faible nombre de pièces entières. Pour calculer le nombre de pointes et de lamelles, les parties distales, discriminantes par définition, ont été ajoutées aux pièces entières. Sur cette base, un total minimum de $\mathbf{5 7 0}$ pointes et 257 lamelles a pu être estimé. Notons que la séparation entre pointes et lamelles s'est effectué sur des critères typologiques stricts ; ont été décomptées comme pointes les seules pièces dont une des extrémités (en général la partie distale) est appointée par des retouches. Certaines lamelles sont néanmoins naturellement pointues.

Que ce soit des pointes ou des lamelles, les armatures à dos possèdent plusieurs caractéristiques qui en font un groupe très homogène. Le dos est toujours rectiligne (5 pièces à dos courbe sur 2413), total et abrupt pour une grande majorité de pièces. La position de la retouche du dos est souvent normale, mais les retouches inverses et croisées sont aussi présentes. L'observation fine de quelques exemplaires suggère que cette retouche est faite à la pression (observations et diagnostic de J. Pélegrin), mais trop peu de pièces ont été examinées pour généraliser cette observation.

Ces armatures peuvent être divisées en plusieurs soustypes:

les pointes (fig. $15: 1-18,22-29$ )

- microgravettes : ce sont les pointes les plus abondantes. Le dos est toujours soigneusement retouché dans sa totalité. II s'incurve dans la partie apicale pour légèrement tronquer l'extrémité et rejoindre l'autre bord. La base est retouchée dans le prolongement du dos et

(12) Les armatures à dos de Saint-Antoine Vitrolles sont actuellement le sujet d'un DEA à l'Université de Provence (URA 164) par C. Montoya. 


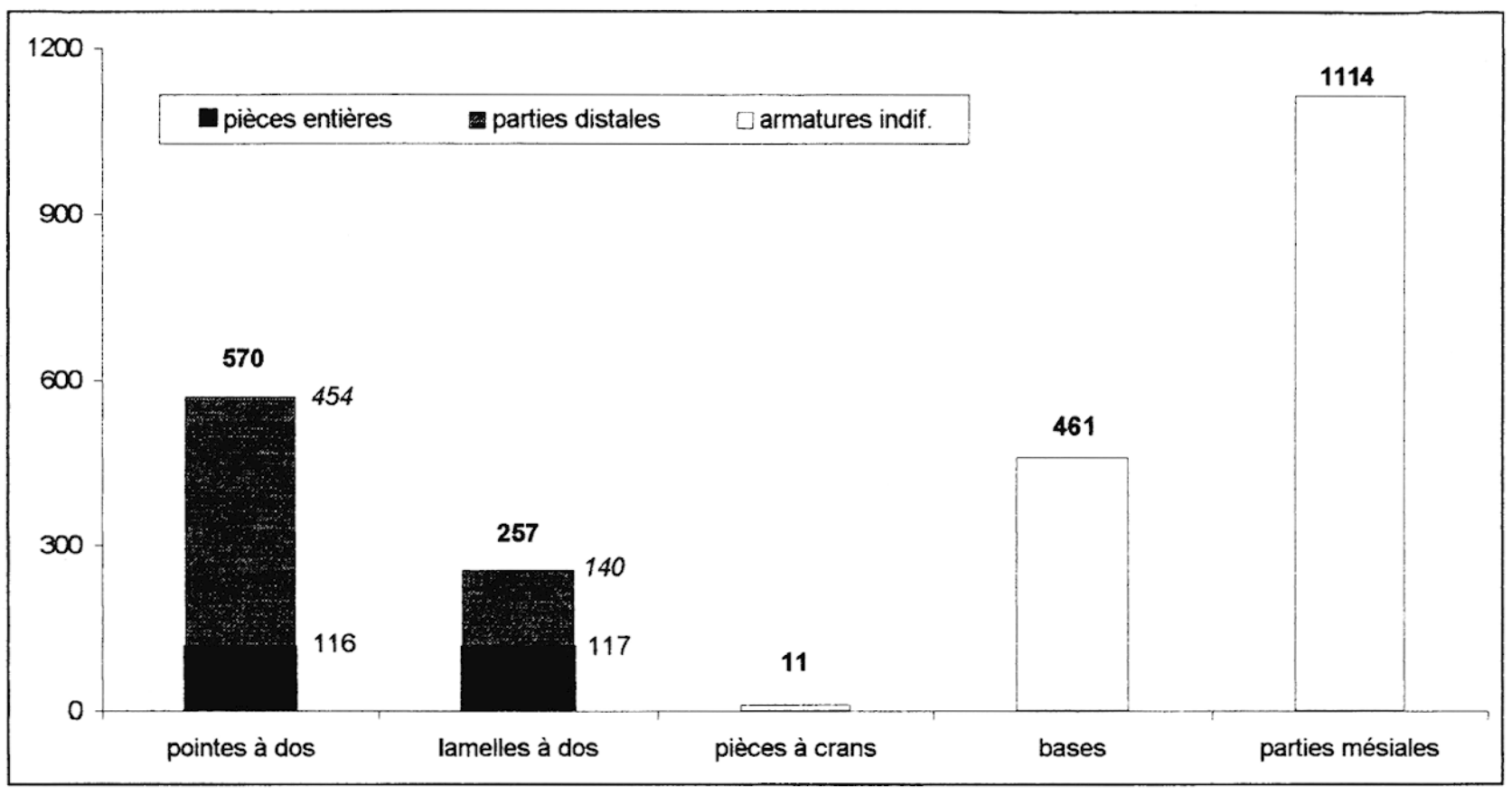

Figure 11 : Saint-Antoine - locus 2

Décompte des armatures à dos $(N=2413)$

Counts of backed projectile points.

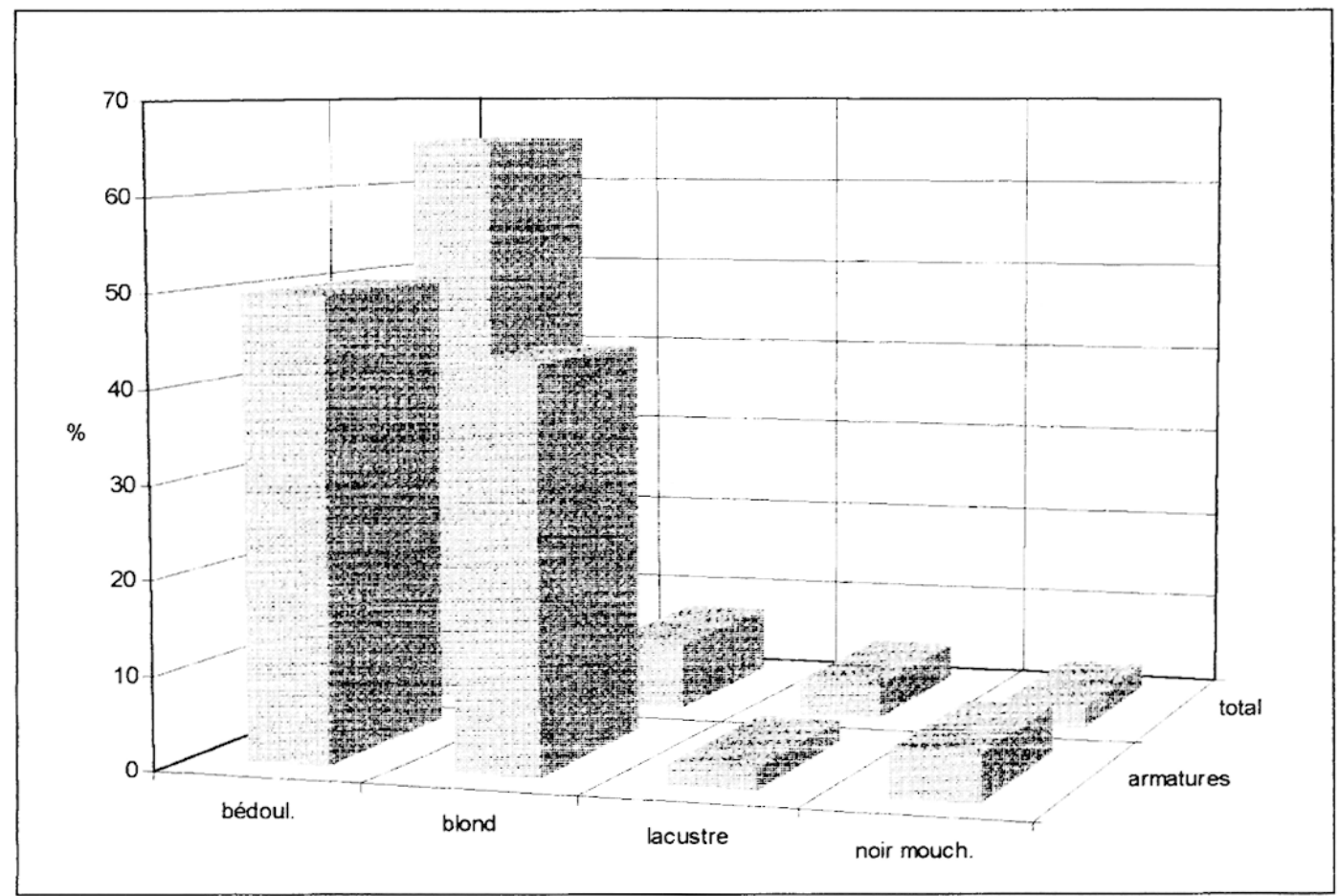

Figure 12 : Saint-Antoine - locus 2

Comparaison (pourcentages) des matières premières des armatures à dos et du total de l'industrie ( $N$ armatures $=177$, $\mathrm{N}$ total $=13596$ )

Comparison (percentages) of raw material used for all the industry and for the backed artefacts 
cette retouche remonte un petit peu sur le bord opposé. A ces caractéristiques qui fondent la définition de la pièce, d'autres éléments peuvent s'ajouter. Le bulbe peut être éliminé par de fines retouches transversales couvrantes qui partent du bord opposé au dos. Cette élimination s'arrête quand son plan horizontal rejoint celui de la face inférieure. Cette élimination peut être éventuellement mise en relation avec les techniques d'emmanchement. La pointe peut elle aussi être reprise par des retouches sub-parallèles couvrantes. L'objectif de cette reprise de l'apex est moins claire. Peut-être s'agitil de redresser l'extrémitë distale pour assurer la meilleure rectitude possible de la pointe ;

- bipointes : peu nombreuses, ces pièces sont parfois difficiles à distinguer des microgravettes. La retouche de la base est très profonde et appointe l'extrémité proximale. Cela confère un aspect un peu courbe au dos en première impression mais en réalité le dos reste véritablement rectiligne dans sa partie mésiale. Cette retouche est systématiquement couplée avec une élimination du bulbe ;

- pointes à dos partiel : quelques pièces ont un dos partiel qui ne se développe que sur le milieu et la partie distale de la lamelle. L'apex peut aussi être repris par des retouches couvrantes comme dans le cas des microgravettes.

les lamelles (fig. $15: 19,21$; fig. $16: 1-6$ )

- lamelles à dos : l'immense majorité des lamelles ne montre pas d'autres aménagements que la réalisation d'un dos rectiligne total. La retouche est toujours profonde, réduisant fortement la largeur de la pièce. La base n'est jamais retouchée ;

- lamelles bi-bords : elles sont rares et toujours confectionnées sur des supports très épais et de dimensions supérieures à la moyenne ;

- lamelles à dos tronquées : elles aussi sont peu abondantes (11 exemplaires). La troncature est toujours unique et en position distale.

les pièces à crans (fig. $16: 32$ )

Ces outils sont très peu nombreux $(N=11)$, toujours fragmentaires et représentés uniquement par les crans. Le dégagement du cran, par retouches abruptes normales, est toujours important, de l'ordre du quart au tiers de la largeur de la pièce quand celle-ci est déterminable. Le débordement de ce dos sur la partie mésiale n'est pas systématique. En l'état, il n'est pas possible de déterminer si ces pièces à crans sont appointées.

\section{Conclusions sur la chaîne opératoire}

De cette première approche de l'industrie lithique ressortent plusieurs éléments, certains assurés, d'autres plus hypothétiques et qui nécessiteront une confirmation ultérieure.

\section{III.1 Un système d'économie du débitage}

La variabilité de certains procédés (supports des nucléus, progression du débitage...) ne doit pas masquer la profonde unité qui régit l'ensemble des processus du débitage et surtout son objectif.

La production est orientée vers l'obtention de lamelles rectilignes détachées par percussion au percuteur de pierre tendre et destinées à être transformées en armatures à dos. Les supports nécessaires à la confection des autres outils, bruts ou retouchés, ne sont pas l'objet d'une production spécifique mais sont prélevés sur les sous-produits de cette chaîne opératoire principale et pratiquement unique (deux nucléus à éclats sont aussi présents).

On peut postuler que cet objectif est présent dès la phase de collecte de la matière première minérale. Le ramassage de volumes de petites dimensions - alors que la reconnaissance des gîtes, notamment bédouliens, a montré que des blocs de grandes dimensions sont présents - peut être mis en relation avec cette production de lamelles. Le débitage d'éclats comme supports de nucléus peut lui aussi être envisagé dans cette même optique. II permet la mise en dimension immédiate des nucléus pour une production lamellaire.

Il est donc possible de rattacher la série de Saint Antoine locus 2 à un système d'économie du débitage selon la définition de C. Perlès (1991), dans lequel une classe d'outil dominante conditionne l'ensemble du débitage. Ce système, d'après Perlès, possède l'avantage "d'une production simultanée de nombreux supports d'outils différents et constitue une économie de temps, d'énergie et de matière première". Notons toutefois que ce schéma général peut subir de légères adaptations. Le silex lacustre par exemple, semble plutôt réservé à une production laminaire que lamellaire. Mais il ne s'agit là probablement que d'un ajustement du débitage lié à la qualité de la matière première.

\section{III.2 Une sélection des supports d'armatures}

S'il ne semble pas y avoir de gestion différenciée des diverses variétés de silex, il est en revanche patent qu'au moment du façonnage une sélection s'est opérée en faveur des lamelles en silex blond. Les raisons en restent inconnues.

Il est possible aussi que les lamelles issues des bord des surfaces de débitage aient été préférées pour la confection des pointes à dos. Mais cette hypothèse demande à être confirmée par une analyse plus poussé des caractéristiques techniques et morphologiques de ces pièces.

\section{III.3 Des procédés de débitage simples...}

Le débitage semble caractérisé par une mise en forme sommaire des surfaces. II est à noter dans ce contexte que l'utilisation du percuteur de pierre tendre permet une plus grande souplesse que le percuteur organique dans la gestion des volumes, notamment un faible carénage 


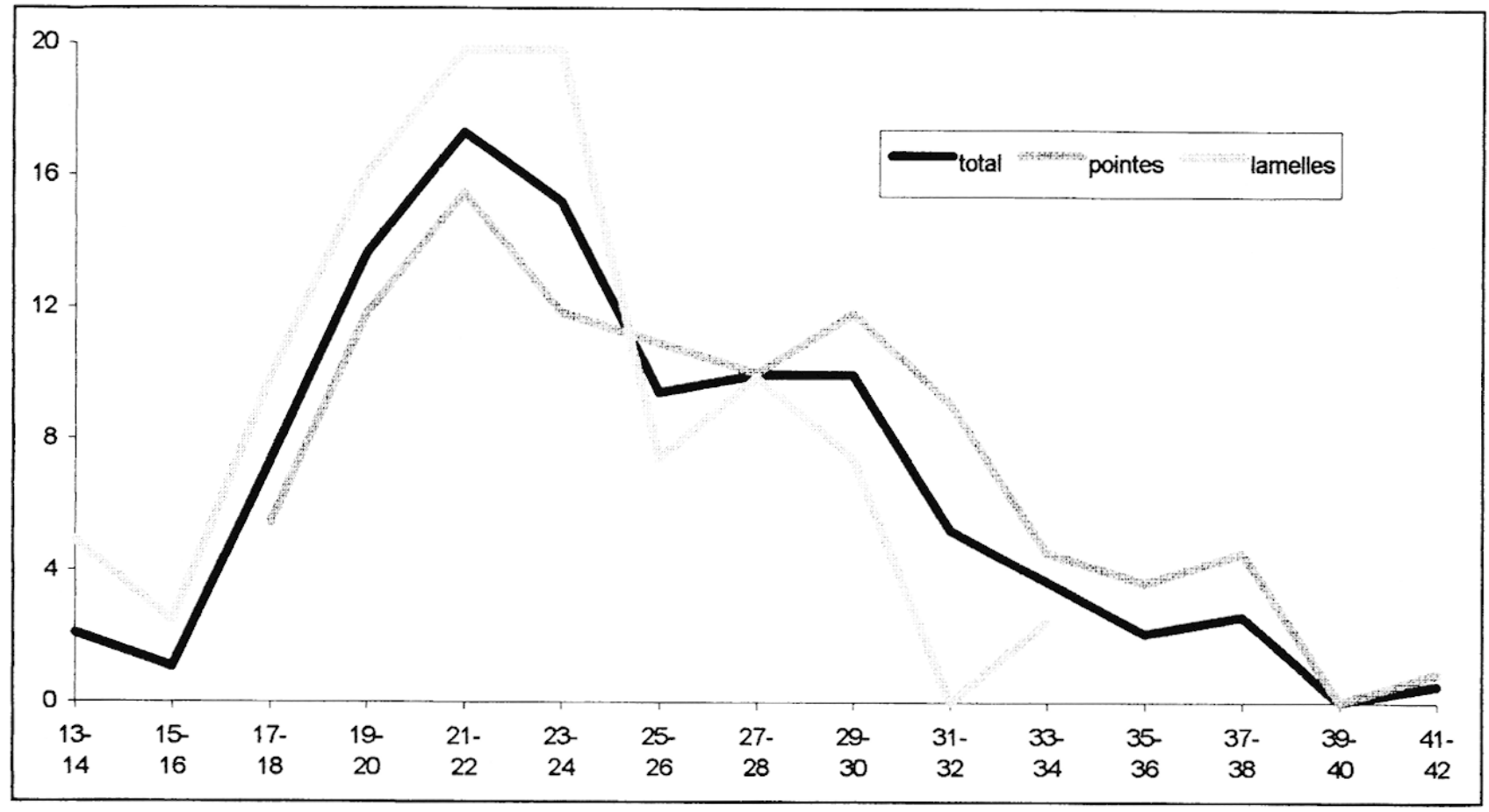

Figure 13 : Saint-Antoine - locus 2

Comparaison des longueurs des pointes et lamelles à dos (pourcentages, classes de $2 \mathrm{~mm}, \mathrm{~N}$ pointes $=110 ; \mathrm{N}$ lamelles $=81$ )

Backed points and backed bladelets length

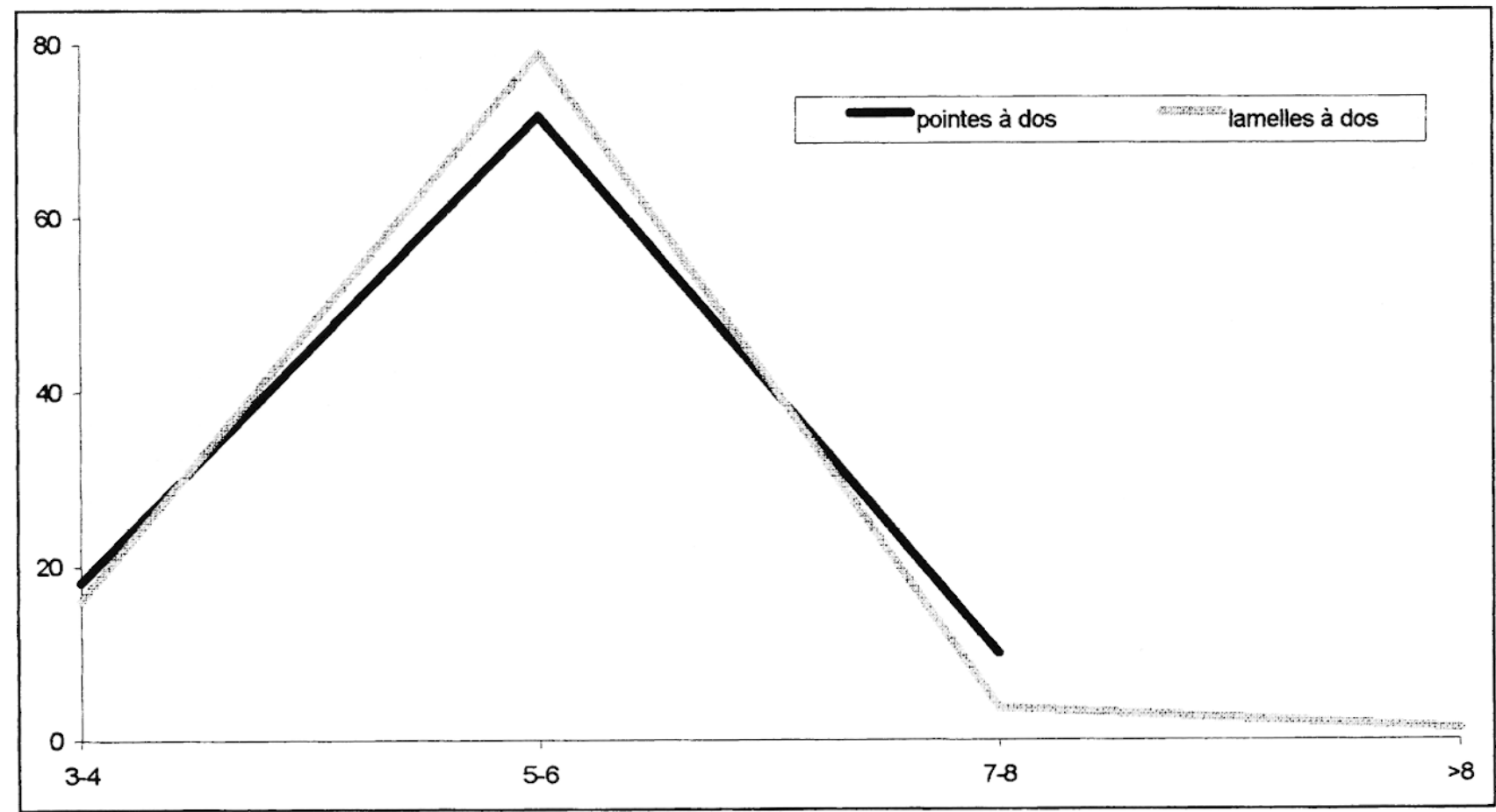

Figure 14 : Saint-Antoine - locus 2

Comparaison des largeurs des pointes et lamelles à dos (classe de $2 \mathrm{~mm}, \mathrm{~N}$ pointes $=110 ; \mathrm{N}$ lamelles $=81$ )

Backed points and backed bladelets width 
initial de la table et un grande amplitude de l'angle plan de frappe/table laminaire. Toutefois, les causalités sont toujours difficiles à établir et il est impossible d'affirmer que l'utilisation du percuteur de pierre tendre est liée à une volonté de simplification de la phase de mise en forme. La même remarque peut être faite en ce qui concerne la rectitude des produits, plus facile à réaliser avec la pierre tendre qu'avec un percuteur tendre.

L'extraction des supports et l'absence de modalités d'entretien fréquentes entraînent un aplatissement rapide des tables laminaires. Quand l'angulation ne peut plus être rattrapée par un ravivage du plan de frappe, la progression du débitage s'effectue alors par une réorientation du nucléus à partir d'un plan de frappe opposé ou d'une surface différente, jointive ou non.

\section{III.4 ... Mais un séquençage de la chaîne opératoire complexe}

La gestion de la matière première lithique témoigne d'un haut degré de prévision. Celle-ci s'exprime en premier lieu dans l'approvisionnement. Deux arguments sont à prendre en considération. D'une part, l'éloignement des gîtes de silex. Sans atteindre des distances considérables (des exemples bien plus lointains sont connus au Paléolithique, cf. par exemple Bracco, 1997 ou FéblotAugustin, 1997), cet éloignement est surtout significatif au vu des quantités transportées. D'autre part, on l'a vu, il semble que le volume des blocs collectés, en taille comme en morphologie, soit sélectionné en fonction des objectifs du débitage.

La quasi-totalité de la séquence de production s'effectue sur place, même si quelques blocs peuvent avoir été décortiqués sur les gîtes. Mais nous avons émis l'hypothèse que certains supports, les lames régulières de plein débitage, ont été emportées au départ (ou aux départs... ?) des groupes humains. Si cette hypothèse est exacte, la gestion de la matière première se déroule dans un temps long, à relier directement aux stratégies d'exploitation des territoires.

Dans tous les cas, l'organisation de la chaîne opératoire lithique de Saint-Antoine - locus 2 montre que les espaces parcourus et leur potentialités en ressources minérales sont connus et maîtrisés. Les solutions mises en cuvre apparaissent parfaitement adaptées ainsi qu'en témoignent les procédés de taille qui ne montrent aucune contrainte d'économie liée à la disponibilité en matière première.

\section{Attribution typologique}

Les caractéristiques de l'industrie lithique du locus 2 de Saint-Antoine permettent de proposer, comme l'on fait Muret et al. (1991) pour le locus 1, un rattachement de cette série à l'Epigravettien évolué ou final, selon les auteurs (cf. (Broglio, sous presse), tel qu'il a été défini par nos collègues italiens. L'absence de toute pointe à dos courbe du type pointe azilienne (ou assimilée à ce groupe, comme les pointes d'lstres, de Malaurie, ...), malgré la grande variabilité typologique de ce groupe d'armatures (Célérier, 1977, 1993), permet d'exclure un rattachement aux complexes aziliens ou même "aziloïdes".

Plusieurs gisements italiques montrent des convergences typologiques nettes avec la série de Vitrolles. Ce sont notamment les importantes séquences de l'abri Tagliente (niveaux supérieur, Bartolomei et al., 1982, 1984) et de l'abri Soman dans le Val d'Adige (Tagliacozzo et Cassoli, 1993). Tous ces niveaux sont datés radiochronologiquement du Bölling/Alleröd au Dryas récent.

Une mention particulière doit être faite pour le site de Val Lastari, situé par radiocarbone dans l'Alleröd et dans lequel l'industrie est très proche de Saint-Antoine (Broglio et al., 1993, Peresani, 1993). Parmi les nucléus lamellaires, les types prismatiques rappellent fortement ceux observés à Vitrolles, même au niveau des phases d'entretien (réfection des tables laminaires par grands enlèvements à partir du plan de frappe). En ce qui concerne l'outillage retouché, de nombreuses pointes à section triangulaire et à dos rectiligne sont présentes, très similaires aux armatures de Saint-Antoine. On note en particulier un taux important de microgravettes, dont plusieurs exemplaires présentent une élimination du bulbe par retouches couvrantes inverses. Dans les deux séries, les géométriques sont anecdotiques. Parmi les différences, soulignons cependant à Val Lastari le fort indice des burins $(18,6 \%$ des outils), des troncatures ( $26 \%$ des outils), des dos et troncatures $(39,2 \%$ des armatures). Toutefois, la vocation spécifique de ce site d'altitude proche de gîtes importants de matières première et qui semble correspondre, au moins en partie, à un faciès d'atelier peut expliquer ces différences.

\section{Conclusions}

La fouille préventive du locus 2 de Saint-Antoine apporte donc de précieuses données pour des contextes géographiques et chrono-culturels encore mal documentés. Au niveau géographique, Saint-Antoine est le premier site paléolithique reconnu dans les Alpes du sud. Son étude permet ainsi l'obtention d'informations capitales sur le (re)peuplement du massif alpin méridional après le Pléniglaciaire. Au niveau chrono-culturel, il s'insère dans un ensemble épigravettien peu abondant dans le sud-est de la France et souvent représenté par des séries lithiques pauvres. L'importance numérique de cette série autorise au contraire une approche typo-technologique détaillée dont nous avons présenté ici les premiers résultats. Ceux-ci mettent en évidence une chaîne opératoire orientée vers la confection d'armatures de projectiles et complétée par la réalisation des outils nécessaires au traitement des carcasses animales. L'intégration des données lithiques avec les premiers résultats des analyses archéozoologiques et spatiales suggère que le locus 2 de Saint-Antoine correspond à des occupations probablement saisonnières (automne) liées à la chasse et à l'exploitation des cervidés. 

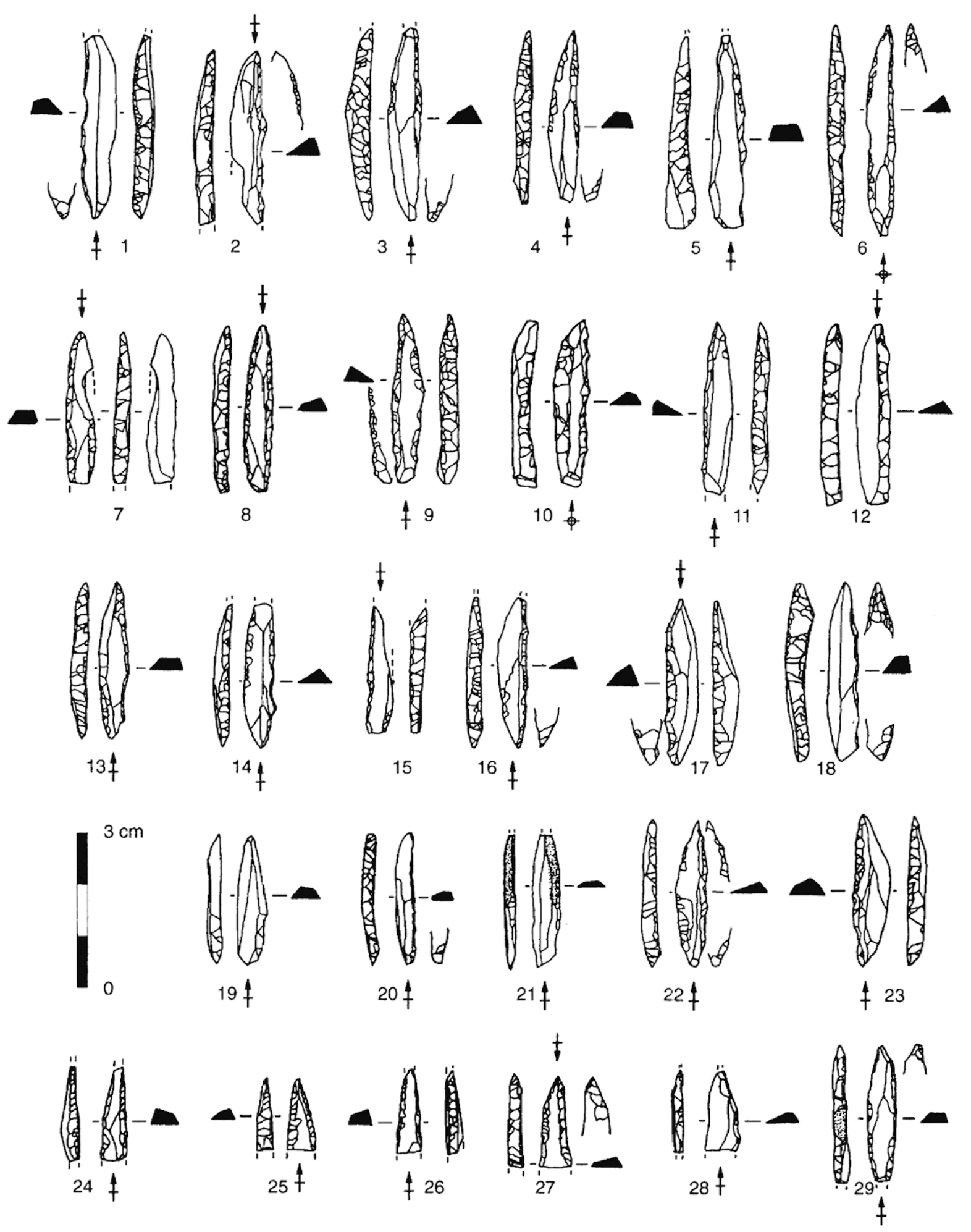

Figure 15 : Saint-Antoine - locus 2, industrie lithique

1-18, 22-29 : pointes à dos, 19-21: lamelles à dos (dessins S. Lancelot, AFAN).

1-18, 22-29 : backed points, $19-21$ : backed bladelets 

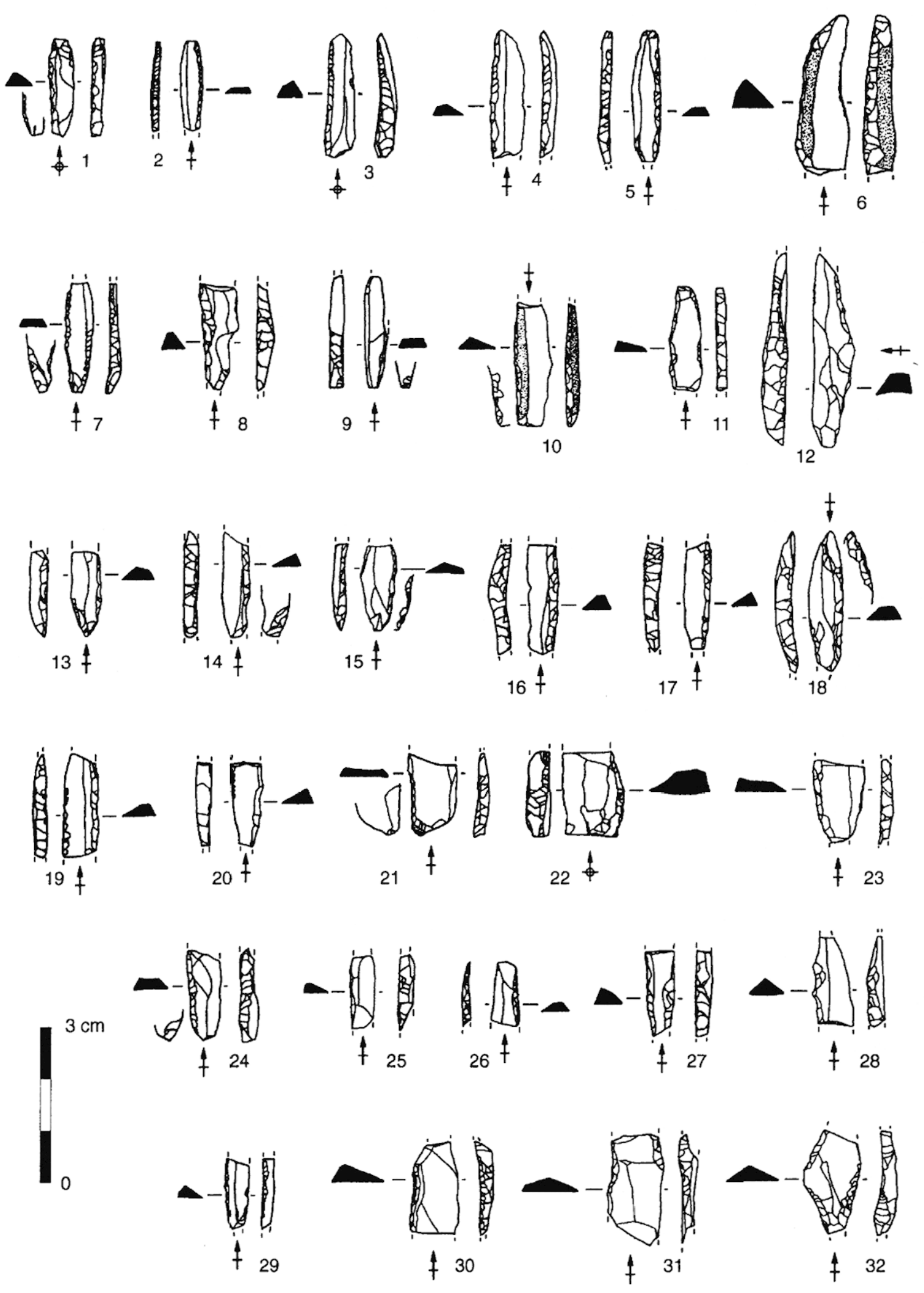

Figure 16 : Saint-Antoine - locus 2, industrie lithique

1-6: lamelles à dos, 7-31: armatures indifférenciées, 32 : pièce à cran (dessins S. Lancelot, AFAN).

1-6 : backed bladelets, 7-31: backed artefacts, 32 : shouldered artefact 


\section{RÉfÉRENCES BIBLIOGRAPHIQUES}

BRACCO J.-P., 1997 - Du site au territoire : l'occupation du sol dans les hautes vallées de la Loire et de l'Allier au Paléolithique supérieur (Massif Central), GalliaPréhistoire, 38 (1996) : 43-67.

BARTOLOMEI G., BROGLIO A., CATTANI L., CRESMACHI M., GUERRESCHI A., MANTOVANI E., PERETTO C. et SALA B., 1982 - I depositi würmiani del Riparo Tagliente, Ann. Univ. Ferrara (N.S.), 15, 3(4) : 61105.

BARTOLOMEI G., BROGLIO A., CATTANI L., CRESMACHI M., GUERRESCHI A., LEONARDI P., et PERETTO C., 1984 - Paleolitico e Mesolitico, Preistoria e Protostoria, 2, Banca Popolare di Verona : 167-319.

BROGLIO A. sous presse - Considérations sur l'Epigravettien italique. Actes du colloque de Banoles : el mundo mediteraneo despues el Pleniglacial.

BROGLIO A., CASTELLETTI L., FRIGO G., MARTELLO G., MASPERO A., ET PERESANI M., 1992 - Le site épigravettien de Val Lastari sur l'Haut Plateau d'Asiago (Préalpes de la Vénétie), Preistoria Alpina - Museo Tridentino di Scienze Naturali, 28, 1 : 207-225.

CANALS I SALOMO A. 1997 - Archéostratigraphie et distribution spatiale, In Gagnepain J., Bracco J.-P., Bidart P. et Vigier S. (Eds.), Saint-Antoine à Vitrolles (HautesAlpes) un site de plein air du Paléolithique supérieur final, Document final de synthèse de fouilles de sauvetage, Ministère de la Culture, Service Régional de l'Archéologie P.A.C.A., AFAN, ESCOTA, Aix-enProvence : 138-134.

CÉLÉRIER G., 1977 - Inventaire morphologique des pointes aziliennes en Périgord : un projet de rationalisation, In Sonneville-Bordes D. (Ed.), La fin des temps glaciaires en Europe, chronostratigraphie et écologie des cultures du Paléolithique final. Actes du Colloque international du CNRS, Bordeaux : 227-238.

CÉLÉRIER G., 1993 - L'abri sous-roche de Pont d'Ambon. Inventaire et typométrie des pointes aziliennes. Gallia-Préhistoire, 35 : 89-98.

FÉBLOT-AUGUSTIN J., 1997 - La circulation des matières premières au Paléolithique. Synthèse des données, perspectives comportementales, Liège, Université de Liège, 2 vol., 275 p. + annexe (Études et Recherches Archéologiques de l'Université de Liège, 75).

GAGNEPAIN J. ET BRACCO J.-P. (Eds.), 1996 - SaintAntoine - locus 1. Site du Paléolithique supérieur final.
Document final de synthèse de fouilles de sauvetage, Ministère de la Culture, Service régional de l'Archéologie PACA, AFAN, Escota, Aix-en-Provence, 97 p.

GAGNEPAIN J., BRACCO J.-P., BIDART P. ET VIGIER S. (Eds.), 1997 - Saint-Antoine à Vitrolles (Hautes-Alpes) un site de plein air du Paléolithique supérieur final, Document final de synthèse de fouilles de sauvetage, Ministère de la Culture, Service Régional de l'Archéologie P.A.C.A., AFAN, ESCOTA, Aix-enProvence, 374 p. + annexes.

MURET A., D'ANNA A., JAUBERT J. et JORDA M. 1991 - Un gisement tardiglaciaire de plein-air dans les Alpes du Sud : Saint-Antoine (Vitrolles, Hautes-Alpes), Bulletin de la Société Préhistorique Française, 88, 2 : 49-57.

PERESANI M., 1993 - Flint exploitation at epigravettian sites in the Asagio Plateau (Venetian Prealps), Preistoria Alpina, Museo Tridentino di Scienze Naturali, 28, 1 : 193205.

PERLĖS C., 1991 - Économie de matières premières et économie du débitage : deux conceptions opposées ? in 25 ans d'Études technologiques en Préhistoire. Bilans et perspectives, Éditions APDCA, Juan-les-Pins : 169-187 (Actes des $\mathrm{XI}^{\circ}$ Rencontres Internationales d'Archéologie et d'Histoire d'Antibes, 1990).

PHILIBERT S., 1997 - Analyse techno-fonctionnelle de l'industrie lithique du site épigravettien de Saint-Antoine, locus 2, In Gagnepain J., Bracco J.-P., Bidart P. et Vigier S. (Eds.), Saint-Antoine à Vitrolles (Hautes-Alpes) un site de plein air du Paléolithique supérieur final, Document final de synthèse de fouilles de sauvetage, Ministère de la Culture, Service Régional de l'Archéologie P.A.C.A., AFAN, ESCOTA, Aix-en-Provence : 208-233.

STOUVENOT C. 1997 - Origine des matières premières minérales. In Gagnepain J., Bracco J.-P., Bidart P. et Vigier S. (Eds.), Saint-Antoine à Vitrolles (Hautes-Alpes) un site de plein air du Paléolithique supérieur final, Document final de synthèse de fouilles de sauvetage, Ministère de la Culture, Service Régional de l'Archéologie P.A.C.A., AFAN, ESCOTA, Aix-enProvence : 124-137.

TAGLIACOZZO A., et CASSOLI P.-F., 1993 - La macrofaune de l'Abri Soman (Val d'Adige, Italie), Preistoria Alpina, Museo Tridentino di Scienze Naturali, 28, 1 : 181192.

TIXIER J., 1963 - Typologie de l'Épipaléolithique du Maghreb, CRAPE, Alger, 209 p. (Mémoire, 2).

Remerciements : Réalisée en contexte de sauvetage urgent, la fouille du locus 2 de Saint-Antoine à bénéficiée d'une collaboration de qualité entre le Service Régional de l'Archéologie PACA, l'AFAN et le maître d'ouvrage, la société des autoroutes Esterel - Côte d'Azur, Provence, Alpes (ESCOTA). Nous tenons à remercier aussi les responsables de la fouille 1988-1990 du locus 1, J. Jaubert, M. Grenet et A. Muret, ainsi que J. Pélegrin et B. Valentin pour leurs remarques pertinentes au sujet de l'industrie lithique. 